\title{
Piper aduncum essential oil: a promising insecticide, acaricide and antiparasitic. A review
}

\author{
Andrea Durofil ${ }^{1,2}$, Matteo Radice ${ }^{1, *}$, José Blanco-Salas ${ }^{2}$, and Trinidad Ruiz-Téllez ${ }^{2}$ \\ ${ }^{1}$ Universidad Estatal Amazónica, Km 21/2 Vía Puyo-Tena, 160150 Puyo, Ecuador \\ ${ }^{2}$ Department of Vegetal Biology, Ecology and Earth Science, Faculty of Sciences, University of Extremadura, 06006 Badajoz, Spain
}

Received 19 November 2020, Accepted 18 April 2021, Published online 3 May 2021

\begin{abstract}
Several studies have assessed the potential of essential oils as substitutes for synthetic pesticides, in order to counter insect resistance to commercial pesticides. Piper aduncum L. is a very common shrub in the Amazon Rainforest and in other subtropical areas. The objective of this review was to analyse the existing information on $P$. aduncum essential oil as a raw material for new bioproducts for sustainable pest disease management. With this review, we collected and critically analysed 59 papers, representing all the studies that aimed to evaluate the essential oil properties of this species as an insecticide, acaricide and antiparasitic. The chemical composition differs depending on the origin, although phenylpropanoid dillapiole is the most cited component, followed by myristicin, 1,8-cineole and $\beta$-ocimene. Between the acaricidal, antiparasitic and synergistic activities, the insecticidal effects are highly promising, with optimal results against the malaria vector Aedes aegypti, with an $\mathrm{LC}_{50}$ that ranges between 57 and $200 \mu \mathrm{g} / \mathrm{mL}$. Acaricidal activity has mainly been reported against Tetranychus urticae, showing an $\mathrm{LC}_{50}$ that ranges between 5.83 and $7.17 \mu \mathrm{g} / \mathrm{mL}$. Antiparasitic activity has predominately been found on Leishmania amazonensis, and antipromastigote activity has been found to be between 23.8 and $25.9 \mu \mathrm{g} / \mathrm{mL}$. Concerning the synergistic effect between dillapiole and synthetic insecticides, four studies on Spodoptera frugiperda found promising results with cypermethrin. In this review, we highlighted the potential of $P$. aduncum essential oil as a biopesticide, also focusing on the lack of information about applied research. We also provide suggestions for future investigations.
\end{abstract}

Key words: Piper aduncum, Essential oil, Insecticide, Acaricide, Antiparasitic, Dillapiole.

Résumé - L'huile essentielle de Piper aduncum : un insecticide, acaricide et antiparasitaire prometteur. Une synthèse. Plusieurs études ont abordé le potentiel des huiles essentielles comme substitut aux pesticides de synthèse, afin de lutter contre la résistance des insectes aux pesticides commerciaux. Piper aduncum L. est un arbuste très commun dans la forêt amazonienne et dans d'autres zones subtropicales. L'objectif de ce travail est d'analyser les informations existantes sur l'huile essentielle de $P$. aduncum comme matière première de nouveaux bioproduits pour une gestion durable des maladies nuisibles. Dans cette synthèse, nous avons collecté et analysé de manière critique 59 articles, représentant toutes les études qui visaient à prouver les propriétés des huiles essentielles de cette espèce en tant qu'insecticide, acaricide et antiparasitaire. La composition chimique diffère selon la provenance, bien que le phénylpropanoïde dillapiole soit le composant le plus cité, suivi de la myristicine, du 1,8-cinéole et du $\beta$-ocimène. À côté des activités acaricide, antiparasitaire et de synergie avec les autres composants, les effets insecticides sont les plus prometteurs, avec des résultats optimaux contre le vecteur du paludisme Aedes aegypti, avec une $\mathrm{CL}_{50}$ comprise entre 57 et $200 \mu \mathrm{g} / \mathrm{mL}$. Une activité acaricide a été principalement rapportée contre Tetranychus urticae avec une $\mathrm{CL}_{50}$ comprise entre 5,83 et 7,17 $\mu \mathrm{g} / \mathrm{mL}$. L'activité antiparasitaire a été montrée principalement sur Leishmania amazonensis et l'activité antipromastigote s'est avérée être comprise entre 23,8 et $25,9 \mu \mathrm{g} / \mathrm{mL}$. Concernant l'effet synergique entre le dillapiole et les insecticides synthétiques, quatre études sur Spodoptera frugiperda ont mis l'accent sur des résultats prometteurs avec la cyperméthrine. Avec cette étude, nous avons mis en évidence le potentiel de l'huile essentielle de P. aduncum en tant que biopesticide, en insistant sur le manque d'informations de la recherche appliquée et sur certaines propositions pour de futures recherches.

\footnotetext{
*Corresponding author: mradice@uea. edu. ec
} 


\section{Introduction}

About $80 \%$ of the world population relies predominantly on plants and plant extracts for health care [81]. Starting from the Orient, mainly from Egypt, Persia and India, through to the Western World, from the ancient Greeks to the Romans, and finding a clear definition in the Middle Ages, Essentials Oils (EOs) are a fundamental part of the history of our civilization [37].

After World War II, there was new trend to mostly study synthetic chemical substances, which led to a decrease in the use of botanical extracts. Clearly, synthetic chemical compounds are more effective compared to natural extracts, but the lack of knowledge about natural substances is too great to compare them fairly: only a small fraction of the 250,000 plant species has been studied properly [91, 102].

Every day, there is increasing evidence about the consequences of using synthetic antiparasitics and insecticides, including their role in environmental pollution, their residual presence in foods and feeds, and most of all their function in developing resistance in parasites and insects [10, 33, 39-41, 85].

The Amazon Rain Forest is one of the most megadiverse places in the world, with more than 50,000 plant species, including at least 14,000 seed plants [13]. For instance, more than 250 species are used by indigenous communities as medicinal treatments in just a small corner of the forest [101].

For the same reason, there are thousands of studies that aim to define the proprieties of the EOs derived from this megadiversity, such as Viola surinamensis [57], Guatteriopsis species [20] and Lippia grandis [89].

Piper species belong to one of the largest genera of basal angiosperm [97] and are widespread in the tropical and subtropical regions of the world. They have a long history of use in traditional medicine and many studies have aimed to prove their efficacy. For instance, EOs from $P$. auritum showed promising results in the inhibition of promastigote proliferation in different species of the Leishmania genus [67], and EOs from P. hispidum showed high antileishmanial activity [43]. Also, $P$. cubeba EOs have anti-trypomastigote and anti-amastigote activity in Trypanosoma cruzi [24]. Various compounds from different species have been studied to assess these activities: dihydrochalcones from $P$. longicaudatum, where asebogenin ( $2^{\prime}, 6^{\prime}, 4$-trihydroxy-4'-methoxydihydrochalcone) showed inhibitory effects against Staphylococcus aureus and methicillinresistant S. aureus (MRSA) [48]; prenylated hydroquinone from $P$. crassinervium with trypanocidal activity [56]; and eupomatenoid-5 isolated from leaves of $P$. regnellii which induces apoptosis in Leishmania amazonensis [36].

In this genus, we also find $P$. aduncum, which has a geographic range that extends mainly through the Neotropics, Southern Asia and the South Pacific [47]. It is a shrub or small tree up to 2-5 (-8) $\mathrm{m}$ tall with pubescent stems. Leaves up to $20 \mathrm{~cm}$ long and 5-9 wide, oblong-elliptic or lanceolate, scabrous on the upper surface and pubescent underneath, acuminate, base rounded or slightly lobed, alternate, distichous. Short petioles rarely up to $8 \mathrm{~cm}$, pubescent. Prophylls up to $25 \mathrm{~mm}$ present. Inflorescence $5-17 \mathrm{~cm}$, forming arching cream to green spikes on peduncles $8-15 \mathrm{~mm}$ long, sparsely pubescent. Floral perianth absent. Androecium with four stamens,

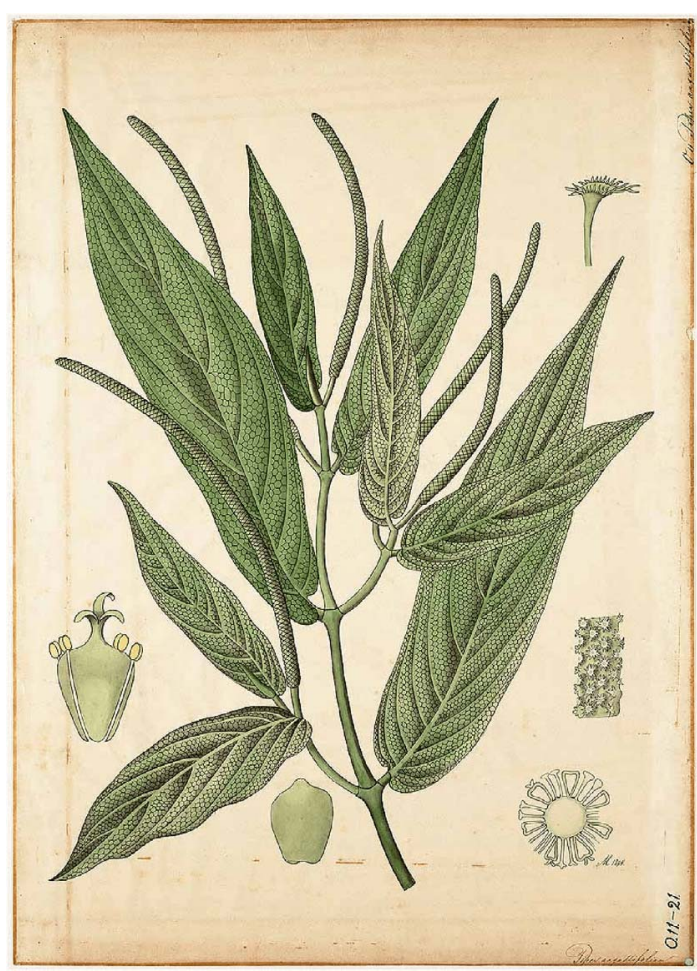

Figure 1. Piper aduncum L. (from Botanischen Wandplatet at http://www.plantillustrations.org/.

anthers $0.2-0.3 \mathrm{~mm}$ long. Floral bracts $0.4-0.7 \mathrm{~mm}$ wide, triangular-round, densely yellow-white ciliate. Ovary with three stigmas. One-seeded berries $0.8-1 \mathrm{~mm}$ wide, obovoid, round from above, glabrous. Seeds reticulated [100] (Fig. 1).

Several ethnobotanical uses have been reported, such as the treatment of inflammation and cuts, skin irritations, "bone pains" and nasal haemorrhage. A tea made from its leaves is used to stop pulmonary haemorrhage, to treat tenesmus in women in labor, or to relieve menstrual colic. It is also mentioned as a folk remedy against kidney disorders, stomach ache, ulcers, rheumatism and skin infections; it is externally applied to relieve skin eruptions in babies. This same tea was described as a diuretic when mixed with corn silk tea. The ethanolic extract obtained from dry leaves was traditionally used to relieve headache and water infusion of buds to lower cholesterol. The entire plant has been mentioned for hot baths in order to help patients during their convalescence; moreover, the tea has been described as a traditional antihemorrhagic and a health tonic [6, 22].

De Castro et al. provided evidence of the effectiveness of cardamonin as a schistosomicidal chalcone from $P$. aduncum extracts, inhibiting the ATP diphosphohydrolase of Schistosoma mansoni, the major aetiological agent of human schistosomiasis [14].

Many of the biological proprieties of $P$. aduncum EO have been studied, for instance its antibacterial and antifungal activity [68], which showed good results against the problematic agents of nosocomial infections such as Staphylococcus aureus, S. epidermidis and S. lentus [9]. Similarly, there are promising results in the prevention of infection in immunocompetent or immunocompromised patients for its activity against Cryptococcus neoformans [69]. There are also antioxidant 
[45], anti-inflammatory [74] and antiplatelet [38] activities, among others.

In order to carry out a systematic review, we collected all the studies regarding insecticidal, acaricidal and antiparasitic activity, mostly aiming to find a way to describe results which can be useful for an alternative or complementary strategy against leishmaniasis and malaria, considering that these diseases are still a great challenge in many countries from both the Eastern and Western tropical and subtropical regions [51].

\section{Materials and methods}

To assess the compound's activities, we selected articles from the electronic databases PubMed (https://pubmed.ncbi. nlm.nih.gov/), SciFinder (https://scifinder.cas.org), ScienceDirect (https://www.sciencedirect.com/), ISI-Web of Science (http://apps.webofknowledge.com), SciELO (https://scielo.org/) and Google Scholar (https://scholar.google.com/). To manage bibliographic references, we used Mendeley software (https:// www.mendeley.com/). Most of the papers date from the last 20 years, but we also included some key data starting from 1948 to develop the introduction. To gather information, we only considered those articles regarding the use of the essential oil in its totality or the compounds derived from it: no semi-synthetic element's activity is shown in this review.

We collected all articles related, on the one hand to antiparasitic activity mainly focusing on the Leishmania genus, and on the other, to insecticide properties, most of all regarding the multiple studies on controlling malaria vectors, among others. And finally, we collected articles on acaricidal activity and the applications of this volatile oil in synergy with already known chemical insecticides.

We chose "essential oil", "insecticide", "acaricide", "antiparasitic" and "dillapiole" as keywords and searched them in different combinations with the main keyword "Piper aduncum".

To form the tables, we selected the following criteria: geographic distribution of the plant used for the extraction, parts of the plant used, method of extraction, main compounds found in the oil, type of application, and organism in which it was applied and activity that it produced in that organism, specifying the concentration used to obtain different ranges of the same activity (when not specified, we reported the effect of the minor time experiments).

\section{Results and discussion \\ Geography and aim of the studies}

Using these criteria, we were able to collect 59 papers, in English, Portuguese and Spanish. As shown in Figure 2, most of the articles aimed to analyse the properties of the EOs of $P$. aduncum plants taken from the Amazon Rain Forest. We counted 39 from the Amazon, whereas only 8 were from the Atlantic Forest, and just 6 from both the Central America region (Cuba) and the Asian Tropics (Malaysia).

Most of these articles focused on insecticidal activity: we considered 43 experiments $(60 \%)$ which aimed to determine

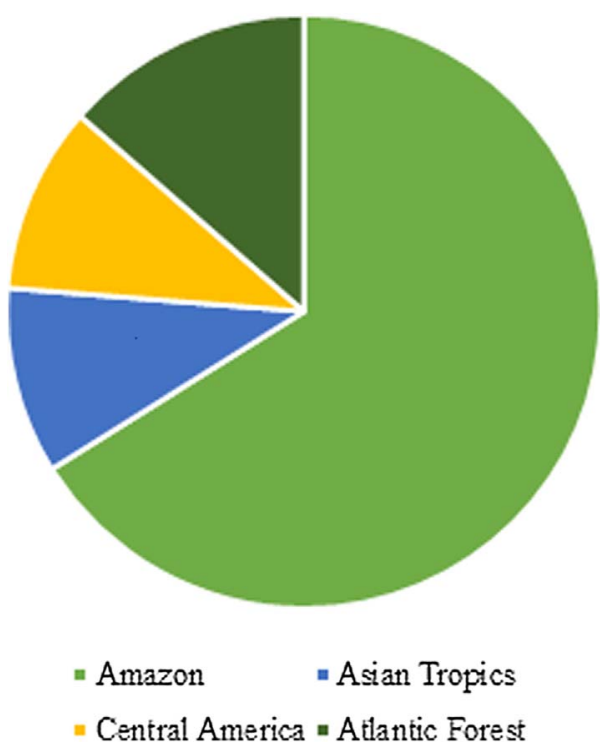

Figure 2. Biogeographic precedence of plant material in the reviewed papers.

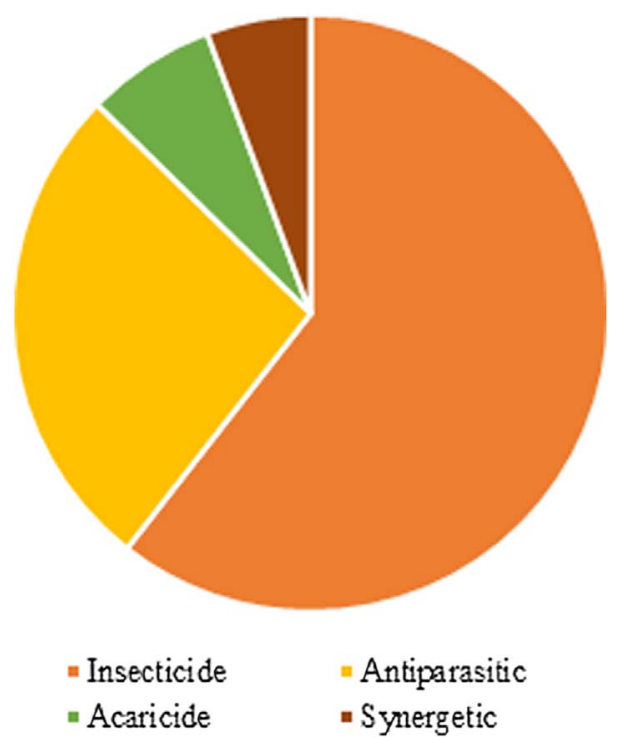

Figure 3. Distribution of the focus of the topics in the revised bibliography.

this type of activity. We also counted 5, 19 and 4 experiments (7, 27 and 6\%) regarding the acaricidal and antiparasitic activities and the synergistic effect as an insecticide, respectively (Fig. 3).

\section{Extraction of the EO and its components}

To obtain the essential oil, the leaves were the part of the plant mostly used in the experiments, sometimes together with fine stems. Regarding extraction methods, hydro-distillation (HD) by the conventional method using a Clevenger type apparatus was the most common, except in the experiments that defined the synergistic effect of the EO, which used steamdistillation (SD) to extract the oil. Gas chromatography was 
Table 1. Chemical structure of the main components of $P$. aduncum essential oil. From the PubChem database (https://pubchem.ncbi.nlm. nih.gov/).

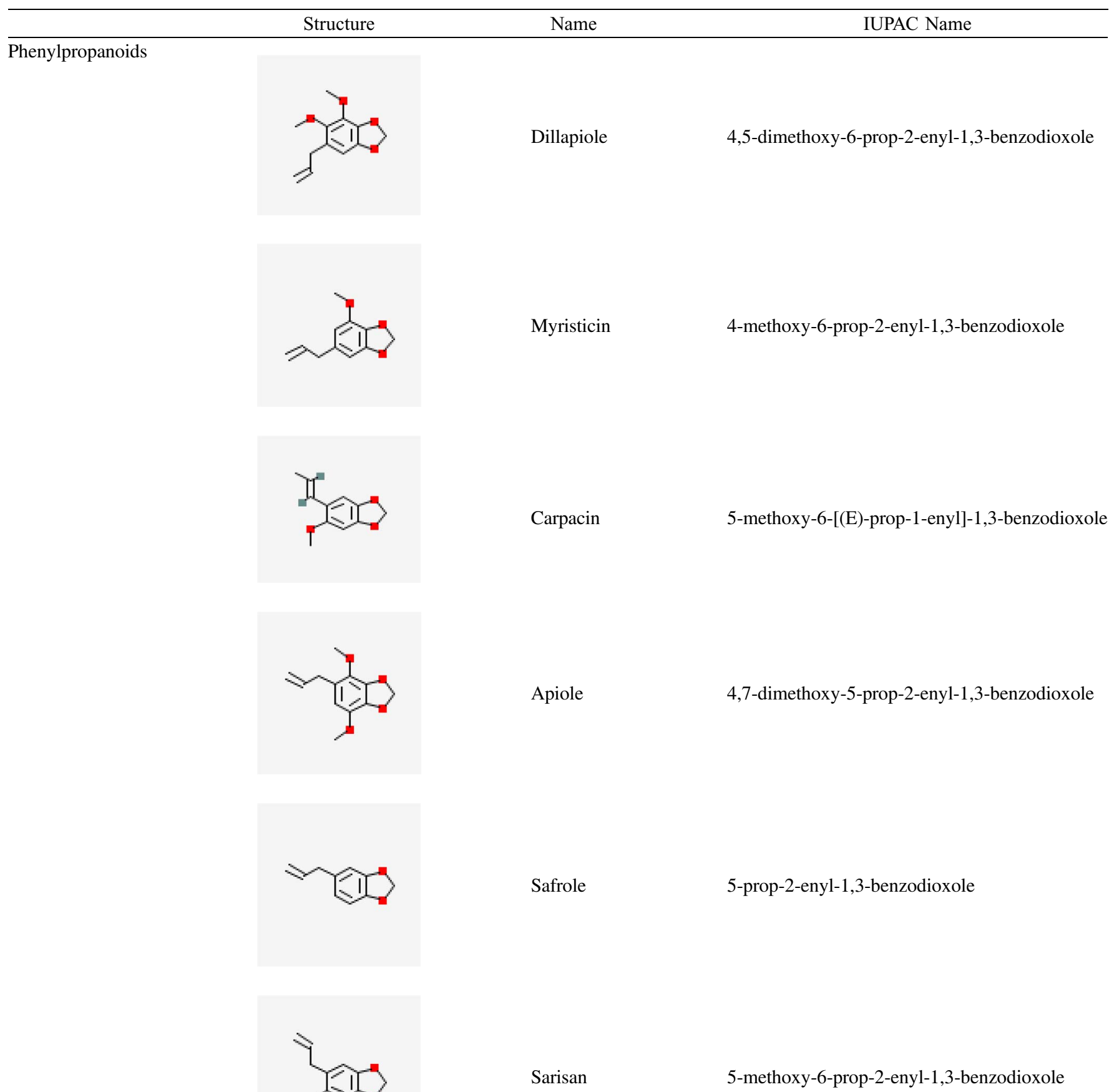


Table 1. (Continued)

Structure
(3E)-3,7-dimethylocta-1,3,6-triene

the basic procedure used, mainly together with mass spectrometry, to analyse and determine its chemical properties.

\section{Compounds of the essential oil}

The chemical composition of the EO in the different studies mainly shows two large groups: phenylpropanoids and monoterpenes (Table 1).

According to Salehi et al. (2019) [84], the Piper genus is known to be a source of essential oils and Piper plants may contain different EOs from several organs and tissues such as seeds, leaves, fruits, branches, stems, and roots; more than 270 identified compounds have been found in Piper species. However, phytochemical studies on essential oils of different Piper species have pointed out high variability in chemical composition. As mentioned by Silva et al. (2017) [93], studies regarding essential oil composition of different Piper species found nine chemotypes characterised by 1,8-cineole, (E)-nerolidol, dillapiole and asaricin. Additionally, due to the resilient nature of the plants, the direct consequence of this biological approach is that the variability of secondary metabolism reflects the adaptation capacity of the plant to all external factors $[16$, 61]. This chemical evidence concerning essential oil variability is highly relevant to identify specific chemotypes that could be useful in the modern food and health products market, even though variable composition is a frequent obstacle to their use. The present review, pointing out the main constituents reported by different research, sheds light on these aspects of variability within the species.

Dillapiole was almost always the main component characterising the oil, followed by myristicin. However, the articles highlight variable abundance mainly due to the different growth conditions and geographical origins, which inevitably affect the qualitative and quantitative profile of the phytocomplex. Dillapiole was the most cited component and the one that had the most promising proprieties, but importantly dillapiole showed better activities as a component of the entire EO than as an isolated compound. Due to this variability, dillapiole amounts ranged from $9.20 \%$ [94] to $94.84 \%$ [87], even though several studies reported that dillapiole represents more than the $70 \%$ of total essential oil composition, as reported in Tables 2-5.

For EOs, variable composition is a frequent condition that makes it difficult to develop standardised batches. The choice and selection of specific chemotypes, the study of the balsamic period of the species and rigorous quality control on extraction, can partially mitigate the problem of variability, allowing production with reproducible quantities of the main components.

Like other phenylpropanoids, dillapiole is a result of the shikimic acid pathway [11] and several authors found biological activities of the isolated molecule or EOs rich in dillapiole. It can be found in a variety of plants, and various studies have aimed to define its uses or functions. These include its gastroprotective function when extracted from Peperomia pellucida [82], and its toxicity against the fungus Leucoagaricus gongylophorus in the control of fungus-feeding ants (Tribe Attini), an agricultural pest in the Neotropics [83]. It is a phenylpropanoid constituted by a benzodioxole with a methyl group in the aromatic ring and an alkyl group in the side chain. The structure of this organic compound is strictly related to its function and activities [74], and when we take a wider view on its synergistic function with some other compounds (Table 1), we observe that this same structure helps to amplify the effects of these chemical substances. This supports its potential when mixed, and not isolated. Finally, other articles cite the activity of semisynthetic derivatives of dillapiole against certain species of the genus Aedes [23, 34], and show its cytotoxic effects against a variety of tumour cells [32].

\section{Insecticidal activity}

In Table 2, we show the results obtained in experiments on the insecticidal properties of the EO. We indicate the scientific names and how the insect is commonly classified. When specified, the concentration of dillapiole ranged between the $8.43 \%$ 
Table 2. Insecticidal activity.

\begin{tabular}{|c|c|c|c|c|c|c|c|}
\hline Country & Part & Extraction & Main compound & Application & Insect & Activity & $\overline{\text { Ref }}$ \\
\hline Brazil & NS & NS & NS & $\begin{array}{l}\text { Contact (contaminated } \\
\text { surface: filter paper } \\
\text { and grain) }\end{array}$ & $\begin{array}{l}\text { Sitophilus zeamais } \\
\text { (Maize weevil) } \\
\text { (Coleoptera) }\end{array}$ & $\begin{array}{l}\text { Adulticide (filter paper: } \mathrm{LC}_{50}=0.6 \mu \mathrm{L} / \mathrm{cm}^{2} \\
\mathrm{LC}_{95}=1.38 \mu \mathrm{L} / \mathrm{cm}^{2} ; \text { grain: } \\
\mathrm{LC}_{50}=0.64 \mu \mathrm{L} / \mathrm{g} \\
\mathrm{LC}_{95}=12.74 \mu \mathrm{L} / \mathrm{g} \text { ) }\end{array}$ & [59] \\
\hline $\begin{array}{l}\text { Brazil, Acre } \\
\text { (Embrapa } \\
\text { Acre) }\end{array}$ & Aerial parts & SD & $\begin{array}{l}\text { Dillapiole }(73.97 \%) \\
\quad \text { safrole }(3.92 \%) \\
\text { sarisan }(2.84 \%)\end{array}$ & $\begin{array}{l}\text { Fumigation; Contact; } \\
\text { Topical }\end{array}$ & $\begin{array}{l}\text { Sitophilus zeamais } \\
\text { (Maize weevil) } \\
\text { (Coleoptera) }\end{array}$ & $\begin{array}{l}\text { Adulticide (fumigation: } \mathrm{LC}_{50}=0.56 \mu \mathrm{L} / \mathrm{g} \text {; } \\
\text { contact: } \mathrm{LC}_{50}=2.87 \mu \mathrm{L} / \mathrm{cm}^{2} ; \text { topical: } \\
\mathrm{LD}_{50}=0.03 \mu \mathrm{L} / \mathrm{g} \text { of insect) }\end{array}$ & [104] \\
\hline $\begin{array}{l}\text { Brazil, Acre } \\
\text { (Embrapa } \\
\text { Acre) }\end{array}$ & Aerial parts & SD & $\begin{array}{l}\text { Dillapiole }(69.3,79.9 \\
\quad \text { and } 85.4 \%)\end{array}$ & $\begin{array}{l}\text { Topical } \\
\text { Residual contact }\end{array}$ & $\begin{array}{l}\text { Diaphorina citri } \\
\quad \text { (Asian citrus psyllid) } \\
\text { (Hemipteran) }\end{array}$ & $\begin{array}{l}\text { Nymphicide (dillapiole } 69.3 \%=95.71 \% \\
\text { mortality; } 79.9 \%=97.14 \% \\
85.4 \%=98.57 \%) \\
\text { Adulticide }(69.3 \%=46.25 \% \\
\quad 79.9 \%=88.75 \% ; 85.4 \%=96.25 \%)\end{array}$ & [106] \\
\hline $\begin{array}{l}\text { Brazil, Acre } \\
\text { (Embrapa } \\
\text { Acre) }\end{array}$ & Aerial parts & SD & $\begin{array}{l}\text { Dillapiole, safrole, } \\
\text { sarisan }\end{array}$ & $\begin{array}{l}\text { Topical; Contact } \\
\text { (contaminated } \\
\text { surface) }\end{array}$ & $\begin{array}{l}\text { Cerotoma } \\
\quad \text { tingomarianus } \\
\text { (Coleoptera) }\end{array}$ & $\begin{array}{l}\text { Adulticide (topical: } \mathrm{LD}_{50}=0.002 \mathrm{~mL} / \mathrm{mg} \\
\text { of insect; contact: } \mathrm{LC}_{50}=0.06 \mathrm{~mL} / \mathrm{cm}^{2} \text { ) }\end{array}$ & {$[26]$} \\
\hline $\begin{array}{l}\text { Brazil, Acre } \\
\text { (Embrapa } \\
\text { Acre) }\end{array}$ & Leaves & HD & NS & $\begin{array}{l}\text { Fumigation; } \\
\text { Repellent }\end{array}$ & $\begin{array}{l}\text { Callosobruchus } \\
\text { maculatus (Cowpea } \\
\text { weevil) (Coleoptera) }\end{array}$ & $\begin{array}{l}\text { Adulticide }\left(\mathrm{LC}_{50}=169.50 \mu \mathrm{L} / \mathrm{L} \text { air }\right) ; \\
\quad \text { Repellence to oviposition }(0.5 \mathrm{~mL} / \mathrm{kg} \\
\text { reduced } 66.06 \%) \\
\text { Ovicide }(68.63 \% \text { at } 0.5 \mathrm{~mL} / \mathrm{kg}) ; \text { Repellence } \\
\quad(\text { attraction reduced } \pm 5 \%)\end{array}$ & {$[72]$} \\
\hline $\begin{array}{l}\text { Brazil, Acre } \\
\text { (Embrapa } \\
\text { Acre) }\end{array}$ & Leaves & HD & Dillapiole (73.97\%) & $\begin{array}{l}\text { Topical; Contact } \\
\text { (contaminated } \\
\text { surface) }\end{array}$ & $\begin{array}{l}\text { Tenebrio molitor } \\
\text { (Mealworm) } \\
\text { (Coleoptera) }\end{array}$ & $\begin{array}{l}\text { Larvicide (topical: } \\
\qquad \mathrm{LD}_{50}=0.009 \mu \mathrm{L} / \mathrm{mg} \text { of insect; contact: } \\
\mathrm{LC}_{50}=0.033 \mu \mathrm{L} / \mathrm{cm}^{2} \text { ) }\end{array}$ & {$[27]$} \\
\hline $\begin{array}{l}\text { Brazil, Acre } \\
\text { (Embrapa } \\
\text { Acre) }\end{array}$ & Leaves & SD & Dillapiole $(71.9 \%)$ & $\begin{array}{l}\text { Topical and residual } \\
\text { contact }\end{array}$ & $\begin{array}{l}\text { Spodoptera frugiperda } \\
\text { (Fall armyworm) } \\
\text { (Lepidoptera) }\end{array}$ & $\begin{array}{l}\text { Larvicide (topical: } \\
\qquad \mathrm{LD}_{50}=1.07 \mu \mathrm{L} / \mathrm{mg} \text { of insect; residual: } \\
\mathrm{LC}_{50}=1169.70 \mathrm{ppm} \text { ) }\end{array}$ & {$[30]$} \\
\hline $\begin{array}{l}\text { Brazil, Acre } \\
\text { (Embrapa } \\
\text { Acre) }\end{array}$ & Leaves & SD & Dillapiole $(71.9 \%)$ & $\begin{array}{l}\text { Topical and residual } \\
\text { contact }\end{array}$ & $\begin{array}{l}\text { Spodoptera frugiperda } \\
\text { (Fall armyworm) } \\
\text { (Lepidoptera) }\end{array}$ & $\begin{array}{l}\text { Larvicide (topical: } \\
\qquad \mathrm{LD}_{50}=0.012 \mu \mathrm{L} / \mathrm{mg} \text { of insect; residual: } \\
\mathrm{LD}_{50}=0.00011 \mu \mathrm{L} / \mathrm{cm}^{2} \text { ) }\end{array}$ & {$[29]$} \\
\hline $\begin{array}{l}\text { Brazil, Acre } \\
\text { (Embrapa } \\
\text { Acre) }\end{array}$ & Leaves & SD & Dillapiole $(71.9 \%)$ & $\begin{array}{l}\text { Topical and residual } \\
\text { contact }\end{array}$ & $\begin{array}{l}\text { Spodoptera frugiperda } \\
\text { (Fall armyworm) } \\
\text { (Lepidoptera) }\end{array}$ & $\begin{array}{l}\text { Larvicide (topical: } \mathrm{LD}_{50}=0.00011 \mu \mathrm{L} / \\
\quad \text { insect; residual: } \mathrm{LC}_{50}=1169.7 \mathrm{ppm} \text { ) }\end{array}$ & {$[28]$} \\
\hline $\begin{array}{l}\text { Brazil, Acre } \\
\text { (Embrapa } \\
\text { Acre) }\end{array}$ & Leaves & SD & Dillapiole (71.9\%) & $\begin{array}{l}\text { Topical and residual } \\
\text { contact }\end{array}$ & $\begin{array}{l}\text { Spodoptera frugiperda } \\
\text { (Fall armyworm) } \\
\text { (Lepidoptera) }\end{array}$ & $\begin{array}{l}\text { Larvicide (topical: } \mathrm{LD}_{50}=0.00011 \mu \mathrm{L} / \mathrm{mg} \\
\text { of insect; residual: } \mathrm{LC}_{50}=0.012 \mu \mathrm{L} / \\
\mathrm{cm}^{2} \text { ) }\end{array}$ & {$[31]$} \\
\hline $\begin{array}{l}\text { Brazil, Acre } \\
\text { (Embrapa } \\
\text { Acre) }\end{array}$ & NS & NS & $\begin{array}{l}\text { Dillapiole }(73.97 \%) \\
\text { safrole }(3.92 \%) \\
\text { sarisan }(2.84 \%)\end{array}$ & Contact & $\begin{array}{l}\text { Callosobruchus } \\
\text { maculatus (Cowpea } \\
\text { weevil) (Coleoptera) }\end{array}$ & $\begin{array}{l}\text { Adulticide }(100 \%=50 \mu \mathrm{L} / 20 \mathrm{~g}) \\
\text { Ovicide }(>90 \%=50 \mu \mathrm{L} / 20 \mathrm{~g}) ; \text { Blocking } \\
\text { egg-hatching }(>90 \%=50 \mu \mathrm{L} / 20 \mathrm{~g})\end{array}$ & {$[77]$} \\
\hline $\begin{array}{l}\text { Brazil, } \\
\quad \text { Amazonas }\end{array}$ & Leaves & HD & *Dillapiole & $\begin{array}{l}\text { Contact (dilution in } \\
\text { water) }\end{array}$ & $\begin{array}{l}\text { Aedes aegypti (Yellow } \\
\text { fever mosquito) } \\
\text { (Diptera) }\end{array}$ & $\begin{array}{l}\text { Larvicide }\left(\mathrm{LC}_{50}=200 \mu \mathrm{g} / \mathrm{mL}\right) \\
\text { Pupaecide }\left(\mathrm{LC}_{50}=200 \mu \mathrm{g} / \mathrm{mL}\right)\end{array}$ & [79] \\
\hline $\begin{array}{l}\text { Brazil, } \\
\text { Amazonas } \\
\text { (INPA) }\end{array}$ & Leaves & HD & *Dillapiole & Contact & $\begin{array}{l}\text { Drosophila } \\
\quad \text { melanogaster (Fruit } \\
\text { fly) (Diptera) }\end{array}$ & $\begin{array}{l}\text { Larvicide }(400 \mu \mathrm{g} / \mathrm{mL}=70 \%, 2000 \mu \mathrm{g} / \\
\mathrm{mL}=100 \%)\end{array}$ & {$[1]$} \\
\hline
\end{tabular}


Table 2. (Continued)

\begin{tabular}{|c|c|c|c|c|c|c|c|}
\hline Country & Part & Extraction & Main compound & Application & Insect & Activity & Ref \\
\hline $\begin{array}{l}\text { Brazil, } \\
\text { Amazonas }\end{array}$ & Leaves & HD & $\begin{array}{l}\text { Dillapiole }(52.37 \%) \\
\gamma \text {-terpinene }(8.98 \%)\end{array}$ & $\begin{array}{l}\text { Contact } \\
\text { Contact (gelatine } \\
\text { nanoparticles) }\end{array}$ & $\begin{array}{l}\text { Cerataphis lataniae } \\
\text { (Palm aphid) } \\
\text { (Hemiptera) }\end{array}$ & $\begin{array}{l}\text { Adulticide }\left(\mathrm{LC}_{50}=219.4 \mu \mathrm{g} / \mathrm{mL}\right. \\
\mathrm{LC}_{90}=864.8 \mu \mathrm{g} / \mathrm{mL} ; \text { loaded } \\
\text { nanoparticles } 500 \mu \mathrm{g} / \mathrm{mL}=90 \%)\end{array}$ & [94] \\
\hline $\begin{array}{l}\text { Brazil, } \\
\text { Amazonas }\end{array}$ & Leaves & HD & $\begin{array}{l}\text { Dillapiole }(52.37 \%) \\
\quad \gamma \text {-terpinene }(8.98 \%)\end{array}$ & $\begin{array}{l}\text { Contact; Contact } \\
\text { (gelatine } \\
\text { nanoparticles) }\end{array}$ & $\begin{array}{l}\text { Aedes aegypti (Yellow } \\
\text { fever mosquito) } \\
\text { (Diptera) }\end{array}$ & $\begin{array}{l}\text { Larvicide }\left(\mathrm{LC}_{50}=68.2 \mu \mathrm{g} / \mathrm{mL}\right. \\
\mathrm{LC}_{90}=125.3 \mu \mathrm{g} / \mathrm{mL} ; \text { loaded } \\
\text { nanoparticles } 500 \mu \mathrm{g} / \mathrm{mL}=100 \%)\end{array}$ & [94] \\
\hline $\begin{array}{l}\text { Brazil, } \\
\text { Amazonas }\end{array}$ & Leaves & SD & *Dillapiole & Contact & $\begin{array}{l}\text { Aedes aegypti (Yellow } \\
\text { fever mosquito) } \\
\text { (Diptera) }\end{array}$ & $\begin{array}{l}\text { Adulticide }\left(\mathrm{LC}_{50}=0.381 \mu \mathrm{L} / \mathrm{cm}^{2}\right. \\
\left.\mathrm{LC}_{90}=0.575 \mu \mathrm{L} / \mathrm{cm}^{2}\right)\end{array}$ & [96] \\
\hline $\begin{array}{l}\text { Brazil, Mato } \\
\text { Groso }\end{array}$ & Leaves & HD & $\begin{array}{l}\text { Myristicin }(30.03 \%), \\
\text { aromadendrene } \\
(9.20 \%), \text { dillapiole } \\
(8.43 \%), \alpha \text {-serinene } \\
(7.31 \%), \text { tridecane } \\
(6.26 \%), \gamma \text {-elemene } \\
(4.58 \%), \text { o-cymene } \\
(4.20 \%)\end{array}$ & Topical & $\begin{array}{l}\text { Euschistus heros } \\
\text { (Brown stink bug) } \\
\text { (Hemiptera) }\end{array}$ & $\begin{array}{l}\text { Adulticide }\left(\mathrm{LD}_{50}=36.23 \mathrm{mg}\right. \\
\left.\mathrm{LD}_{90}=50.42 \mathrm{mg}\right)\end{array}$ & [19] \\
\hline $\begin{array}{l}\text { Brazil, Mato } \\
\text { Groso }\end{array}$ & Leaves & HD & $\begin{array}{l}\text { Myristicin, } \\
\text { isomyristicin, } \\
\text { asaricim, dillapiole, } \\
\text { isocroweacin }\end{array}$ & Ingestion; Topical & $\begin{array}{l}\text { Helicoverpa armigera } \\
\text { (Cotton bollworm) } \\
\text { (Lepidoptera) }\end{array}$ & $\begin{array}{l}\text { Larvicide (ingestion: } 1^{\text {st }} \text { instar } \\
\qquad \mathrm{LT}_{50}=<14.20 \text { days, } 3^{\text {rd }} \\
\mathrm{LT}_{50}=<16.89 ; \text { topical: } 1^{\mathrm{st}} \\
\mathrm{LT}_{50}=<14.68,3^{\text {rd }} \mathrm{LT}_{50}=<10.73 \text { ) }\end{array}$ & [88] \\
\hline $\begin{array}{l}\text { Brazil, Mato } \\
\text { Groso }\end{array}$ & Leaves & HD & $\begin{array}{l}\text { Myristicin }(30.03 \%), \\
\text { aromadendrene } \\
(9.20 \%), \text { dillapiole } \\
(8.43 \%), \alpha \text {-serine } \\
(7.31 \%), \text { tridecane } \\
(6.26 \%), \gamma \text {-elemene } \\
(4.58), \text { o-cymene } \\
(4.20 \%)\end{array}$ & $\begin{array}{l}\text { Contact and Immersion } \\
\text { (eggs); Topical }\end{array}$ & $\begin{array}{l}\text { Euschistus heros } \\
\quad \text { (Brown stink bug) } \\
\text { (Hemiptera) }\end{array}$ & $\begin{array}{l}\text { Ovicide (Immersion: } \\
\mathrm{LC}_{50}=15.64 \mathrm{mg} / \mathrm{mL} ; \text { Contact: } \\
\left.\mathrm{LC}_{50}=24.29 \mathrm{mg} / \mathrm{mL}\right) \\
\left.{\text { Nymphicide }\left(\mathrm{LD}_{50}=11.37 \mathrm{mg} / \mathrm{mL}\right.} \mathrm{LD}_{90}=38.95 \mathrm{mg} / \mathrm{mL}\right) \\
\text { Adulticide }\left(>20 \mathrm{mg} / \mathrm{mL}: \mathrm{LT}_{50}=<6\right. \\
\text { days) }\end{array}$ & [103] \\
\hline $\begin{array}{l}\text { Brazil, Mato } \\
\text { Groso }\end{array}$ & Leaves & SD & $\begin{array}{l}\text { Dillapiole, z-carpacin, } \\
\text { myristicin }\end{array}$ & Topical & $\begin{array}{l}\text { Tibraca limbativentris } \\
\text { (Stink bug) } \\
\text { (Hemiptera) }\end{array}$ & Ovicide $\left(\mathrm{LC}_{50}=2.499 \%\right)$ & [49] \\
\hline $\begin{array}{l}\text { Brazil, Mato } \\
\text { Groso }\end{array}$ & Leaves & SD & $\begin{array}{l}\text { Dillapiole, myristicin, } \\
\text { z-carpacin }\end{array}$ & Topical; Ingestion & $\begin{array}{l}\text { Chrysodeixis includens } \\
\text { (Soybean looper) } \\
\text { (Lepidoptera) }\end{array}$ & $\begin{array}{l}\text { Larvicide (at } 24 \mathrm{~h} \text { : ingestion: } \\
\qquad \mathrm{LC}_{50}=3.5 \%, \mathrm{LC}_{90}=6.2 \% \\
\text { topical: } \mathrm{LC}_{50}=16.2 \% \\
\mathrm{LC}_{90}=30.0 \% \text { ) }\end{array}$ & [86] \\
\hline $\begin{array}{l}\text { Brazil, Minas } \\
\text { Gerais }\end{array}$ & Leaves & HD & $\begin{array}{l}\text { 1,8-cineole }(53.9 \%), \\
\alpha \text {-pinene }(12.7 \%), \\
\beta \text {-pinene }(8.5 \%), \\
\text { trans-ocimene } \\
(5.7 \%)\end{array}$ & $\begin{array}{l}\text { Fumigation (dilution in } \\
\text { water) }\end{array}$ & $\begin{array}{l}\text { Aedes aegypti (Yellow } \\
\text { fever mosquito) } \\
\text { (Diptera) }\end{array}$ & $\begin{array}{l}\text { Larvicide }(250 \mathrm{ppm}=40 \%, \\
500 \mathrm{ppm}=100 \%)\end{array}$ & [70] \\
\hline Brazil, Pará & Aerial parts & HD & Dillapiole (64. 4\%) & $\begin{array}{l}\text { Contact (contaminated } \\
\text { surface) }\end{array}$ & $\begin{array}{l}\text { Solenopsis saevissima } \\
\quad \text { (Fire ant) } \\
\text { (Hymenoptera) }\end{array}$ & Adulticide $\left(\mathrm{LC}_{50}=58.4 \mathrm{mg} / \mathrm{L}\right)$ & [99] \\
\hline
\end{tabular}


Table 2. (Continued)

\begin{tabular}{|c|c|c|c|c|c|c|c|}
\hline Country & Part & Extraction & Main compound & Application & Insect & Activity & Ref \\
\hline Brazil, Pará & Leaves & HD & $\begin{array}{l}\text { Asaricine, myristicin, } \\
\text { dillapiole, (E)- } \\
\beta \text {-ocimene, } \\
\text { piperitone }\end{array}$ & Spraying & $\begin{array}{l}\text { Anticarsia gemmatalis } \\
\text { (Velvetbean } \\
\text { caterpillar) } \\
\text { (Lepidoptera) }\end{array}$ & $\begin{array}{l}\text { Ovicidal }\left(\mathrm{LC}_{50}=1.9 \%\right. \\
\left.\operatorname{LC}_{90}=2.8 \%\right)\end{array}$ & {$[50]$} \\
\hline Brazil, Pará & NS & NS & $\begin{array}{l}\text { Dillapiole }(73.97 \%), \\
\text { safrole }(3.92 \%), \\
\text { sarisan }(2.84 \%)\end{array}$ & Topical & $\begin{array}{l}\text { Callosobruchus } \\
\text { maculatus (Cowpea } \\
\text { weevil) (Coleoptera) }\end{array}$ & $\begin{array}{l}\text { Ovicide }(100 \%=0.5 \mathrm{~L} / \mathrm{t}) ; \text { Block } \\
\quad \text { egg-hatching }(100 \%=0.5 \mathrm{l} / \mathrm{t})\end{array}$ & [76] \\
\hline Brazil, Paraiba & Dried fruit & HD & $\begin{array}{l}\beta \text {-pinene }(32.7 \%), \\
\text { E-caryophyllene } \\
(17.1 \%)\end{array}$ & $\begin{array}{l}\text { Contact (dilution in } \\
\text { water) }\end{array}$ & $\begin{array}{l}\text { Aedes aegypti (Yellow } \\
\text { fever mosquito) } \\
\text { (Diptera) }\end{array}$ & Larvicide $\left(\mathrm{LC}_{50}=30.2 \mu \mathrm{g} / \mathrm{mL}\right)$ & [21] \\
\hline $\begin{array}{l}\text { Brazil, } \\
\quad \text { Rondonia }\end{array}$ & Leaves & HD & $\begin{array}{l}\text { (E)-isocroweacin } \\
(29.52 \%) \text { apiole } \\
(28.62 \%) \text { elemicin } \\
(7.82 \%)\end{array}$ & $\begin{array}{l}\text { Fumigation (dilution in } \\
\text { water) }\end{array}$ & $\begin{array}{l}\text { Aedes aegypti (Yellow } \\
\text { fever mosquito) } \\
\text { (Diptera) }\end{array}$ & $\begin{array}{l}\text { Larvicide }\left(\mathrm{LC}_{50}=46 \mathrm{ppm}\right. \\
\mathrm{LC}_{90}=156 \mathrm{ppm} \\
100 \%=500 \mathrm{ppm})\end{array}$ & [87] \\
\hline $\begin{array}{l}\text { Colombia, } \\
\text { Chocó }\end{array}$ & Leaves & HD & $\begin{array}{l}\text { Dillapiole }(48,2 \%) \\
\quad 1,8 \text { cineole }(11,4 \%)\end{array}$ & $\begin{array}{l}\text { Contact (contaminated } \\
\text { surface) }\end{array}$ & $\begin{array}{l}\text { Triblium castaneum } \\
\quad \text { (Red flour beetle) } \\
\text { (Coleoptera) }\end{array}$ & $\begin{array}{l}\text { Repellence }\left(1 \mu \mathrm{L} / \mathrm{cm}^{2}=99 \% \text { for }\right. \\
2 \mathrm{~h})\end{array}$ & [46] \\
\hline $\begin{array}{l}\text { Cuba, La } \\
\text { Habana }\end{array}$ & Leaves & HD & Dillapiole $(82.0 \%)$ & $\begin{array}{l}\text { Fumigation (dilution in } \\
\text { water) }\end{array}$ & $\begin{array}{l}\text { Aedes aegypti (Yellow } \\
\text { fever mosquito) } \\
\text { (Diptera) }\end{array}$ & $\begin{array}{l}\text { Larvicide }\left(\mathrm{LC}_{50}=57 \mathrm{mg} / \mathrm{L}\right. \\
\left.\quad \mathrm{LC}_{90} 5=75 \mathrm{mg} / \mathrm{L}\right)\end{array}$ & [52] \\
\hline $\begin{array}{l}\text { Cuba, La } \\
\text { Habana }\end{array}$ & NS & $\mathrm{SD}$ & NS & $\begin{array}{l}\text { Fumigation (dilution in } \\
\text { water) }\end{array}$ & $\begin{array}{l}\text { Aedes aegypti (Yellow } \\
\text { fever mosquito) } \\
\text { (Diptera) }\end{array}$ & $\begin{array}{l}\text { Larvicide }\left(\mathrm{LC}_{50}=36.0 \mathrm{mg} / \mathrm{mL}\right) \\
\text { Adulticide }(60 \mathrm{mg} / \mathrm{mL} \\
\left.\mathrm{LT}_{50}=0.19 \mathrm{~h}\right)\end{array}$ & [60] \\
\hline $\begin{array}{l}\text { Cuba, La } \\
\text { Habana }\end{array}$ & NS & SD & NS & $\begin{array}{l}\text { Fumigation (dilution in } \\
\text { water) }\end{array}$ & $\begin{array}{l}\text { Aedes aegypti (Yellow } \\
\text { fever mosquito) } \\
\text { (Diptera) }\end{array}$ & $\begin{array}{l}\text { Larvicide }\left(\mathrm{LC}_{50}=35.3 \mathrm{mg} / \mathrm{mL}\right) \\
\text { Adulticide }(30 \mathrm{mg} / \mathrm{mL} \\
\left.\mathrm{LT}_{50}=0.15 \mathrm{~h}\right)\end{array}$ & {$[60]$} \\
\hline $\begin{array}{l}\text { Cuba, La } \\
\text { Habana }\end{array}$ & NS & SD & NS & $\begin{array}{l}\text { Fumigation (dilution in } \\
\text { water) }\end{array}$ & $\begin{array}{l}\text { Aedes aegypti (Yellow } \\
\text { fever mosquito) } \\
\text { (Diptera) }\end{array}$ & $\begin{array}{l}\text { Larvicide }\left(\mathrm{LC}_{50}=57.3 \mathrm{mg} / \mathrm{mL}\right) \\
\text { Adulticide }(40 \mathrm{mg} / \mathrm{mL} \\
\left.\mathrm{LT}_{50}=0.19 \mathrm{~h}\right)\end{array}$ & [60] \\
\hline $\begin{array}{l}\text { Cuba, La } \\
\text { Habana }\end{array}$ & NS & SD & NS & $\begin{array}{l}\text { Fumigation (dilution in } \\
\text { water) }\end{array}$ & $\begin{array}{l}\text { Culex quinquefasciatus } \\
\text { (Southern house } \\
\text { mosquito) (Diptera) }\end{array}$ & $\begin{array}{l}\text { Larvicide }\left(\mathrm{LC}_{50}=59.5 \mathrm{mg} / \mathrm{mL}\right) \\
\text { Adulticide }\left(60 \mathrm{mg} / \mathrm{mL} \mathrm{LT}_{50}=\right. \\
0.17 \mathrm{~h})\end{array}$ & [60] \\
\hline Cuba, La Palma & NS & HD & NS & Topical & $\begin{array}{l}\text { Musca domestica } \\
\text { (Housefly) (Diptera) }\end{array}$ & $\begin{array}{l}\text { Adulticide }\left(\mathrm{LC}_{50}=0.04 \%\right. \\
\left.\quad \mathrm{LC}_{95}=0.33 \%\right)\end{array}$ & [53] \\
\hline $\begin{array}{l}\text { Ecuador, } \\
\text { Pastaza, }\end{array}$ & Aerial parts & HD & $\begin{array}{l}\text { Dillapiole }(48.2 \%), \\
\quad \text { trans-ocimene } \\
(7.5 \%), \\
\beta \text {-caryophyllene } \\
(17.0 \%)\end{array}$ & $\begin{array}{l}\text { Fumigation (dilution in } \\
\text { water) }\end{array}$ & $\begin{array}{l}\text { Aedes aegypti (Yellow } \\
\text { fever mosquito) } \\
\text { (Diptera) }\end{array}$ & $\begin{array}{l}\text { Larvicide }\left(\mathrm{LC}_{50}=23.73 \mathrm{ppm}\right. \\
\quad \mathrm{LC}_{90}=35.51 \mathrm{ppm} ; \mathrm{LC}_{99}= \\
49.31 \mathrm{ppm})\end{array}$ & [90] \\
\hline
\end{tabular}


Table 2. (Continued)

\begin{tabular}{|c|c|c|c|c|c|c|c|}
\hline Country & Part & Extraction & Main compound & Application & Insect & Activity & Ref \\
\hline Malaysia & NS & HD & $\begin{array}{l}\text { (E)- } \beta \text {-ocimene, trans- } \\
\text { caryophyllene, } \\
\text { (z)- } \beta \text { - ocimene, } \\
\beta \text {-pinene, } \alpha \text {-pinene, } \\
\text { germacrena-D, } \\
\text { piperitone, } \\
\gamma \text {-terpinene, } \\
\text { limonene }\end{array}$ & Contact & $\begin{array}{l}\text { Periplaneta americana } \\
\text { (American } \\
\text { cockroach) } \\
\text { (Blattodea) }\end{array}$ & $\begin{array}{l}\text { Adulticide }(80,000 \mathrm{ppm} \text {; females } \\
\mathrm{LC}_{50}=5.31 \mathrm{~h}, \mathrm{LT}_{90}=14.9 \mathrm{~h}, \\
\text { males } \mathrm{LT}_{50}=2.08 \mathrm{~h}, \\
\left.\mathrm{LT}_{90}=5.14 \mathrm{~h}\right) \\
\text { Nymphicide }(80,000 \mathrm{ppm} \\
\left.\mathrm{LT}_{50}=4.68 \mathrm{~h}, \mathrm{LT}_{90}=28.71\right)\end{array}$ & [54] \\
\hline $\begin{array}{l}\text { Malaysia, } \\
\text { Selangor }\end{array}$ & Leaves & $\mathrm{HD} * * *$ & $\begin{array}{l}\text { apiole }(38.01 \%), \\
\text { methyl isobutyl } \\
\text { ketone }(8.26 \%), \\
\text { piperitone }(3.34 \%), \\
\text { caryophyllene } \\
(2.45 \%)\end{array}$ & $\begin{array}{l}\text { Topical (on human } \\
\text { body) }\end{array}$ & $\begin{array}{l}\text { Aedes aegypti (Yellow } \\
\text { fever mosquito) } \\
\text { (Diptera) }\end{array}$ & $\begin{array}{l}\text { Repellence to human bodies ( }>65 \% \\
\text { at } 4 \mathrm{~h} \text { post-application) }\end{array}$ & [58] \\
\hline $\begin{array}{l}\text { Malaysia, } \\
\text { Selangor }\end{array}$ & Leaves & HD & NS & Spraying & $\begin{array}{l}\text { Aedes aegypti (Yellow } \\
\text { fever mosquito) } \\
\text { (Diptera) }\end{array}$ & $\begin{array}{l}\text { Adulticide }\left(\mathrm{LC}_{50}=5.6 \%\right. \\
\left.\qquad \mathrm{LC}_{90}=12.3 \%\right)\end{array}$ & [63] \\
\hline $\begin{array}{l}\text { Malaysia, } \\
\text { Selangor }\end{array}$ & Leaves & HD & NS & Spraying & $\begin{array}{l}\text { Aedes aegypti (Yellow } \\
\text { fever mosquito) } \\
\text { (Diptera) }\end{array}$ & $\begin{array}{l}\text { Adulticide }\left(\mathrm{LC}_{50}=5.5 \%\right. \\
\left.\quad \mathrm{LC}_{90}=12.7 \%\right)\end{array}$ & {$[63]$} \\
\hline $\begin{array}{l}\text { Malaysia, } \\
\text { Selangor }\end{array}$ & Leaves & $\mathrm{HD} * *$ & NS & Topical (human body) & $\begin{array}{l}\text { Aedes aegypti (Yellow } \\
\text { fever mosquito) } \\
\text { (Diptera) }\end{array}$ & $\begin{array}{l}\text { Repellence to human bodies } \\
\qquad\left(\mathrm{ED}_{50}=0.4 \% ; \mathrm{ED}_{90} 5=1.7 \%\right)\end{array}$ & [42] \\
\hline $\begin{array}{l}\text { Malaysia, } \\
\text { Selangor }\end{array}$ & Leaves & HD & NS & Topical (human body) & $\begin{array}{l}\text { Aedes aegypti (Yellow } \\
\text { fever mosquito) } \\
\text { (Diptera) }\end{array}$ & $\begin{array}{l}\text { Repellence to human bodies }\left(60^{\prime \prime}\right. \\
\left.\text { exposure: } \mathrm{ED}=1.5 \mathrm{~g} / \mathrm{cm}^{2}\right)\end{array}$ & [65] \\
\hline $\begin{array}{l}\text { Malaysia, } \\
\text { Selangor }\end{array}$ & NS & HD & NS & Topical (human body) & $\begin{array}{l}\text { Aedes aegypti (Yellow } \\
\text { fever mosquito) } \\
\text { (Diptera) }\end{array}$ & $\begin{array}{l}\text { Repellence to human bodies }\left(90^{\prime \prime}\right. \\
\text { exposure: } \mathrm{ED}_{50}=1.95 \mu \mathrm{g} / \mathrm{cm}^{2} \\
\left.\mathrm{ED}_{90}=18.1 \mu \mathrm{g} / \mathrm{cm}^{2}\right)\end{array}$ & [64] \\
\hline NS & NS & SD & *Dillapiole & $\begin{array}{l}\text { Contact; Residual } \\
\text { contact }\end{array}$ & $\begin{array}{l}\text { Leptinotarsa } \\
\text { decemlineata } \\
\text { (Colorado potato } \\
\text { beetle) (Coleoptera) }\end{array}$ & Larvicide $(0.1 \mathrm{ppm}=92 \%)$ & [55] \\
\hline
\end{tabular}

* Only the main compound was tested;

** Ointment, cream and gel;

*** Dried over anhydrous magnesium sulphate then formulated into Carbopol 934 hydrogels, Aerial parts: Leaves and Branches, HD: hydro-distillation, SD: steam-distillation, NS: not specified, EO: essential oil, $\mathrm{LC}_{50}$ : lethal concentration for $50 \%, \mathrm{LC}_{90}$ : lethal concentration for $90 \%, \mathrm{LD}_{50}$ : lethal dose for $50 \%, \mathrm{LD}_{90}$ : lethal dose for $90 \%$, $\mathrm{LT}_{50}$ : lethal time for $50 \%$, $\mathrm{LT}_{90}$ : lethal time for $90 \%, \mathrm{ED}_{50}$ : effective dose for $50 \%, \mathrm{ED}_{90}$ : effective dose for $90 \%, \mathrm{ED}_{95}$ : effective dose for $95 \%$. 
Table 3. Acaricidal activity.

\begin{tabular}{|c|c|c|c|c|c|c|c|}
\hline Country & Part(s) & Extraction & Main Compound(s) & Application(s) & Mite & Activity(ies) & Ref \\
\hline $\begin{array}{l}\text { Brazil, Amazonas } \\
\text { (Ducke Reserve) }\end{array}$ & Leaves & $\begin{array}{c}\text { Hexane } \\
\text { extract }+ \text { HD }\end{array}$ & Dillapiole $(94.84 \%)$ & Immersion & $\begin{array}{l}\text { Tick (Rhipicephalus } \\
\text { microplus) }\end{array}$ & Larvicide $(0.1 \mathrm{mg} / \mathrm{mL}=100 \%)$ & {$[92]$} \\
\hline Brazil, Amazonas & Leaves & HD & $\begin{array}{l}\text { Dillapiole }(52.37 \%), \\
\quad \gamma \text {-terpinene }(8.98 \%)\end{array}$ & $\begin{array}{l}\text { Contact Contact } \\
\text { (gelatine } \\
\text { nanoparticles) }\end{array}$ & $\begin{array}{l}\text { Mite (Tetranychus } \\
\text { urticae) }\end{array}$ & $\begin{array}{l}\text { Adulticide }\left(\mathrm{LC}_{50}=56.5 \mu \mathrm{g} / \mathrm{mL}\right. \\
\mathrm{LC}_{90}=84.3 \mu \mathrm{g} / \mathrm{mL} ; \text { loaded } \\
\text { nanoparticles } 500 \mu \mathrm{g} / \\
\mathrm{mL}=100 \%)\end{array}$ & [94] \\
\hline Brazil, Pernambuco & Leaves & HD & Dillapiole $(76.5 \%)$ & $\begin{array}{l}\text { Fumigation Residual } \\
\text { contact }\end{array}$ & $\begin{array}{l}\text { Mite (Tetranychus } \\
\text { urticae) }\end{array}$ & $\begin{array}{l}\text { Adulticide (fumigation: } \\
\mathrm{LC}_{50}=0.008 \mu \mathrm{l} / \mathrm{L} \text { air; } \\
\text { residual: } \mathrm{LC}_{50}=5.83 \mu \mathrm{L} / \mathrm{mL} \text { ) } \\
\text { Repellence to oviposition } \\
\text { (fumigation: } 0.001 \mu \mathrm{L} / \mathrm{L} \\
\text { air }=40 \% ; \text { residual: } \\
0.0001 \mu \mathrm{L} / \mathrm{mL}=30 \% \text { ) }\end{array}$ & [4] \\
\hline Brazil, Pernambuco & Leaves & HD & $\begin{array}{l}\text { Dillapiole (28\%), } \\
\quad \alpha \text {-humulene }(1.6 \%), \\
\text { (E)-nerolidol } \\
(0.07 \%) \\
\beta \text {-caryophyllene } \\
(0.21 \%)\end{array}$ & Fumigation Contact & $\begin{array}{l}\text { Mite (Tetranychus } \\
\text { urticae) }\end{array}$ & $\begin{array}{l}\text { Adulticide (Fumigation } \\
\mathrm{LC}_{50}=0.01 \mu \mathrm{L} / \mathrm{L} \text { air; Contact } \\
\left.\mathrm{LC}_{50}=7.17 \mu \mathrm{L} / \mathrm{mL}\right) \\
\text { Repellence }\left(\mathrm{RC}_{50}=0.04 \mu \mathrm{L} /\right. \\
\mathrm{mL})\end{array}$ & [3] \\
\hline Cuba, La Habana & Leaves & HD & $\begin{array}{l}\text { Camphene, camphor, } \\
\text { piperitone, } \\
\text { viridiflorol }\end{array}$ & Contact & $\begin{array}{r}\text { Mite (Varroa } \\
\text { destructor) }\end{array}$ & $\begin{array}{l}\text { Adulticide }(25 \mu \mathrm{L} / \text { Petri } \\
\quad \text { dish }=100 \%)\end{array}$ & [78] \\
\hline
\end{tabular}

$\mathrm{HD}$ : hydro-distillation, $\mathrm{LC}_{50}$ : lethal concentration for $50 \%, \mathrm{LC}_{90}$ : lethal concentration for $90 \%, \mathrm{RC}_{50}$ : repellent concentration for $50 \%$. 
Table 4. Antiparasitic activity.

\begin{tabular}{|c|c|c|c|c|c|c|c|}
\hline Country & Part(s) & Extraction & Main compound(s) & Application(s) & Parasite & Activity(ies) & Ref \\
\hline Brazil, São Paulo & Leaves & HD & Dillapiole & Immersion & $\begin{array}{l}\text { Leishmania } \\
\text { amazonensis } \\
\text { (Euglenozoa) }\end{array}$ & $\begin{array}{l}\text { Leishmanicidal - growth inhibition of } \\
\text { promastigote }\left(\mathrm{IC}_{50}=59.4 \mu \mathrm{m}\right)\end{array}$ & {$[73]$} \\
\hline Brazil, Santa Catarina & Leaves & HD & $\begin{array}{l}\text { (Z)- } \beta \text {-ocimene (7\%), } \\
\text { (E)- } \beta \text {-ocimene } \\
\text { (13.9), safrole } \\
(6.2 \%), \alpha \text {-humulene } \\
(4.9 \%), \alpha \text {-humulene } \\
(20.9 \%), \gamma \text {-cadinene } \\
(5.5 \%), \text { spathulenol } \\
(5.3 \%)\end{array}$ & Incubation & $\begin{array}{l}\text { Leishmania } \\
\quad \text { amazonensis } \\
\quad \text { (Euglenozoa) }\end{array}$ & $\begin{array}{l}\text { Leishmanicidal }- \text { Antipromastigote } \\
\left(\mathrm{IC}_{50}=25.9 \mu \mathrm{g} / \mathrm{mL}\right) \text { and } \\
\text { antiamastigote }\left(\mathrm{IC}_{50}=36.2 \mu \mathrm{g} / \mathrm{mL}\right) \\
\text { activity }\end{array}$ & [7] \\
\hline Cuba, La Habana & Leaves & HD & $\begin{array}{l}\text { Piperitone }(23.7 \%) \\
\quad \text { camphor }(17.1 \%) \\
\text { viridiflorol }(14.5 \%)\end{array}$ & Incubation & $\begin{array}{l}\text { Leishmania } \\
\quad \text { amazonensis } \\
\text { (Euglenozoa) }\end{array}$ & $\begin{array}{l}\text { Leishmanicidal - Antipromastigote } \\
\quad \text { activity }\left(\mathrm{IC}_{50}=23.8 \mu \mathrm{g} / \mathrm{mL}\right)\end{array}$ & {$[68]$} \\
\hline $\begin{array}{l}\text { Brazil, Minas Gerais } \\
\text { (UFLA) }\end{array}$ & Leaves & HD & Nerolidol & Incubation & $\begin{array}{l}\text { Leishmania } \\
\quad \text { braziliensis } \\
\quad \text { (Euglenozoa) }\end{array}$ & $\begin{array}{l}\text { Leishmanicidal }- \text { Antipromastigote } \\
\quad \text { activity }\left(\mathrm{IC}_{50} / 24 \mathrm{~h}=77.9 \mu \mathrm{g} / \mathrm{mL}\right)\end{array}$ & [105] \\
\hline Brazil, São Paulo & Leaves & HD & Dillapiole & Immersion & $\begin{array}{l}\text { Leishmania } \\
\quad \text { braziliensis } \\
\quad \text { (Euglenozoa) }\end{array}$ & $\begin{array}{l}\text { Leishmanicidal }- \text { growth inhibition of } \\
\text { promastigote }\left(\mathrm{IC}_{50}=69.3 \mu \mathrm{m}\right)\end{array}$ & [73] \\
\hline Cuba, La Habana & Aerial parts & SD & NS & Incubation & $\begin{array}{l}\text { Leishmania } \\
\quad \text { braziliensis } \\
\quad \text { (Euglenozoa) }\end{array}$ & $\begin{array}{l}\text { Leishmanicidal - Antipromastigote } \\
\text { activity }(50.8 \mu \mathrm{g} / \mathrm{mL}=100 \%)\end{array}$ & [66] \\
\hline- & - & - & $*$ Dillapiole & Incubation & $\begin{array}{l}\text { Leishmania chagasi } \\
\quad \text { (Euglenozoa) }\end{array}$ & $\begin{array}{l}\text { Leishmanicidal - Antipromastigote } \\
\text { activity }(50 \mu \mathrm{g} / \mathrm{mL}=99 \%)\end{array}$ & [25] \\
\hline Cuba, La Habana & Leaves & HD & $\begin{array}{l}\text { Piperitone }(23.7 \%) \\
\text { camphor }(17.1 \%) \\
\text { viridiflorol }(14.5 \%)\end{array}$ & Incubation & $\begin{array}{l}\text { Leishmania donovani } \\
\quad \text { (Euglenozoa) }\end{array}$ & $\begin{array}{l}\text { Leishmanicidal - Antipromastigote } \\
\text { activity }\left(\mathrm{IC}_{50}=7.7 \mu \mathrm{g} / \mathrm{mL}\right)\end{array}$ & {$[68]$} \\
\hline Cuba, La Habana & Leaves & HD & $\begin{array}{l}\text { Piperitone }(23.7 \%) \\
\quad \text { camphor }(17.1 \%) \\
\text { viridiflorol }(14.5 \%)\end{array}$ & Incubation & $\begin{array}{l}\text { Leishmania infantum } \\
\text { (Euglenozoa) }\end{array}$ & $\begin{array}{l}\text { Leishmanicidal - Antiamastigote } \\
\quad \text { activity }\left(\mathrm{IC}_{50}=8.1 \mu \mathrm{g} / \mathrm{mL}\right)\end{array}$ & {$[68]$} \\
\hline Cuba, La Habana & Leaves & HD & $\begin{array}{l}\text { Piperitone }(23.7 \%) \\
\quad \text { camphor }(17.1 \%) \\
\text { viridiflorol }(14.5 \%)\end{array}$ & Incubation & $\begin{array}{l}\text { Trypanosoma brucei } \\
\quad \text { (Euglenozoa) }\end{array}$ & $\begin{array}{l}\text { Antitrypanosomal activity } \\
\quad\left(\mathrm{IC}_{50}=2.0 \mu \mathrm{g} / \mathrm{mL}\right)\end{array}$ & {$[68]$} \\
\hline $\begin{array}{l}\text { Brazil, Minas Gerais } \\
\quad \text { (UFLA) }\end{array}$ & Leaves & HD & Linalool, nerolidol & Incubation & $\begin{array}{l}\text { Trypanosoma cruzi } \\
\text { (Euglenozoa) }\end{array}$ & $\begin{array}{l}\text { Antitrypanosomal }- \text { activity against } \\
\text { epimastigote } \\
\left(\mathrm{IC}_{50} / 24 \mathrm{~h}=84.7 \mu \mathrm{g} / \mathrm{mL} \text { at } 28{ }^{\circ} \mathrm{C}\right) \text {, } \\
\text { amastigote }\left(\mathrm{IC}_{50} / 24 \mathrm{~h}=9 \mu \mathrm{g} / \mathrm{mL} \text { at }\right. \\
\left.37{ }^{\circ} \mathrm{C}\right) \text {, cell-derived trypomastigote } \\
\left(\mathrm{IC}_{50} / 24 \mathrm{~h}=2.8 \text { and } 3.8 \mu \mathrm{g} / \mathrm{mL} \text { at }\right. \\
28{ }^{\circ} \mathrm{C} \text { and } 4{ }^{\circ} \mathrm{C} \text {, respectively) and } \\
\text { metacyclic trypomastigote } \\
\left(\mathrm{IC}_{50} / 24 \mathrm{~h}=12.1 \mu \mathrm{g} / \mathrm{mL} \text { at } 28{ }^{\circ} \mathrm{C}\right)\end{array}$ & [15] \\
\hline
\end{tabular}


Table 4. (Continued)

\begin{tabular}{|c|c|c|c|c|c|c|c|}
\hline Country & $\operatorname{Part}(\mathrm{s})$ & Extraction & Main compound(s) & Application(s) & Parasite & Activity(ies) & Ref \\
\hline Cuba, La Habana & Leaves & HD & $\begin{array}{l}\text { Piperitone }(23.7 \%) \\
\quad \text { camphor }(17.1 \%) \\
\text { viridiflorol }(14.5 \%)\end{array}$ & Incubation & $\begin{array}{l}\text { Trypanosoma cruzi } \\
\text { (Euglenozoa) }\end{array}$ & $\begin{array}{l}\text { Antitrypanosomal activity } \\
\quad\left(\mathrm{IC}_{50}=2.1 \mu \mathrm{g} / \mathrm{mL}\right)\end{array}$ & [68] \\
\hline Cuba, La Habana & Aerial parts & SD & NS & Incubation & $\begin{array}{l}\text { Trichomonas vaginalis } \\
\text { (Metamonada) }\end{array}$ & $\begin{array}{l}\text { Trichomonacide } \\
\qquad(100 \mu \mathrm{g} / \mathrm{mL}=100 \%)\end{array}$ & [66] \\
\hline Brazil, Ceará & $\begin{array}{l}\text { Aerial parts } \\
\text { (leaves and } \\
\text { branches) }\end{array}$ & SD & $\begin{array}{l}\text { Dillapiole }(76.5 \%), \\
\text { piperitone }(6.1 \%), \\
\text { terpinen-4-ol } \\
(2.3 \%), \text { myristicin } \\
(2.1 \%),(\mathrm{E}) \text { - } \\
\text { caryophyllene } \\
(1.5 \%), \gamma \text {-terpinene } \\
(1.3 \%), \text { germacrene- } \\
\text { D }(1.2 \%), \text { apiole } \\
(1.2 \%)\end{array}$ & Contact & $\begin{array}{l}\text { Plasmodium } \\
\quad \text { falciparum } \\
\text { (Apicomplexa) }\end{array}$ & $\begin{array}{l}\text { Antiplasmodial activity } \\
\quad(72 \mathrm{~h} \text { exposure, } \\
\text { W2: } 1.30 \mathrm{ng} / \mathrm{mL}=100 \% \text {; } \\
\text { Dd2: } 10.30 \mathrm{mg} / \mathrm{mL}=77 \%)\end{array}$ & [62] \\
\hline Cuba, La Habana & Leaves & HD & $\begin{array}{l}\text { Piperitone }(23.7 \%), \\
\text { camphor }(17.1 \%), \\
\text { viridiflorol }(14.5 \%)\end{array}$ & Incubation & $\begin{array}{l}\text { Plasmodium } \\
\quad \text { falciparum } \\
\text { (Apicomplexa) }\end{array}$ & $\begin{array}{l}\text { Antiplasmodial activity } \\
\quad\left(\mathrm{IC}_{50}=1.3 \mu \mathrm{g} / \mathrm{mL}\right)\end{array}$ & [68] \\
\hline Brazil, Amazonas & Leaves & HD & dillapiole $(76.2 \%)$ & Contact & $\begin{array}{l}\text { Haemonchus contortus } \\
\text { (Nematoda) }\end{array}$ & $\begin{array}{l}\text { Egg-hatching inhibition } \\
\left.\text { (IC } C_{50}=5.72 \mathrm{mg} / \mathrm{mL}\right) \\
\text { Blocking larvae development } \\
\left(\mathrm{IC}_{50}=0.10 \mathrm{mg} / \mathrm{mL}\right. \\
\left.\mathrm{IC}_{90}=0.34 \mathrm{mg} / \mathrm{mL}\right)\end{array}$ & [35] \\
\hline Brazil, Minas Gerais & Leaves & HD & $\begin{array}{l}\text { 1,8-cineole }(55.8 \%), \alpha \text { - } \\
\text { terpineol }(5.9 \%), \\
\text { trans-ocimene } \\
(4.8 \%), \beta \text {-pinene } \\
(4.7 \%), \alpha \text {-pinene } \\
(4.5 \%), \\
\text { bicyclogermacrene } \\
(4.4 \%)\end{array}$ & Contact & $\begin{array}{l}\text { Haemonchus contortus } \\
\text { (Nematoda) }\end{array}$ & $\begin{array}{l}\text { Egg-hatching inhibition } \\
\qquad\left(\mathrm{LC}_{90}=8.9 \mathrm{mg} / \mathrm{mL}\right)\end{array}$ & [71] \\
\hline Brazil, Amazonas & Leaves & SD & Dillapiole (92\%) & $\begin{array}{l}\text { Contact (ingestion of } \\
\text { medicated food by } \\
\text { fishes) }\end{array}$ & $\begin{array}{l}\text { Hysterothylacium sp. } \\
\text { (Nematoda) }\end{array}$ & $\begin{array}{l}\text { Larvicide }(64 \mathrm{~mL} / \mathrm{kg}=76.21 \% \\
\text { at } 15 \text { days treatment })\end{array}$ & [18] \\
\hline
\end{tabular}

*Only the main compound was tested, Aerial parts: leaves and branches, HD: hydro-distillation, SD: steam-distillation, NS: not specified, EO: essential oil, LC 90 : lethal concentration for $90 \%, \mathrm{IC}_{50}$ : inhibitory concentration for $50 \%, \mathrm{IC}_{90}$ : inhibitory concentration for $90 \%$. 
Table 5. Synergetic properties of dillapiole along with chemical insecticides.

\begin{tabular}{|c|c|c|c|c|c|c|c|}
\hline Country & Part & Extraction & Main compound & Application & Insect & Activity & Ref \\
\hline $\begin{array}{l}\text { Brazil, } \\
\text { Acre }\end{array}$ & Leaves & SD & $\begin{array}{l}\text { dillapiole } \\
(71.9 \%)+(\text { cypermethrin, } \\
\text { zeta-cypermethrin, permethrin, } \\
\text { esfenvarelate })\end{array}$ & $\begin{array}{l}\text { Topical and } \\
\text { residual } \\
\text { contact }\end{array}$ & $\begin{array}{l}\text { Spodoptera frugiperda } \\
\text { (Fall armyworm) } \\
\text { (Lepidoptera) }\end{array}$ & $\begin{array}{l}\text { Larvicide (topical: } 0.54 \mu \mathrm{L} \text { EO: }+0.0553 \mu \mathrm{L} \text { Cypermethrin } \\
\mathrm{LD}_{50}=0.0093 \mu \mathrm{L} / \mathrm{mg} \text { of insect; }+0.000733 \mu \mathrm{L} \text { Zeta- } \\
\text { Cypermethrin } \mathrm{LD}_{50}=0.00017 \mu \mathrm{L} / \mathrm{mg} ;+0.000327 \mu \mathrm{L} \\
\text { Permethrin } \mathrm{LD}_{50}=0.000068 \mu \mathrm{L} / \mathrm{mg} ;+0.2 \mu \mathrm{L} \text { Esfenvarelate } \\
\mathrm{LD}_{50}=0.053 \mu \mathrm{L} / \mathrm{mg} \text { )(residual: } 584.9 \mathrm{ppm} \text { EO: }+256.70 \mathrm{ppm} \\
\text { Cypermethrin } \mathrm{LC}_{50}=3.52 \mathrm{ppm} ;+747.80 \mathrm{ppm} \text { Zeta- } \\
\text { Cypermethrin } \mathrm{LC}_{50}=617.00 \mathrm{ppm} ;+246.20 \mathrm{ppm} \text { Permethrin } \\
\mathrm{LC}_{50}=14.30 \mathrm{ppm} ;+48756.10 \mathrm{ppm} \text { Esfenvarelate } \\
\left.\mathrm{LC}_{50}=3640.70 \mathrm{ppm}\right)\end{array}$ & $\overline{[30]}$ \\
\hline $\begin{array}{l}\text { Brazil, } \\
\text { Acre }\end{array}$ & Leaves & SD & $\begin{array}{l}\text { dillapiole }(71.9 \%)+ \\
\text { ( } \alpha \text {-cypermethrin, } \\
\beta \text {-cypermethrin, fenpropathrin, } \\
\gamma \text {-cyhalothrin) }\end{array}$ & $\begin{array}{l}\text { Topical and } \\
\text { residual } \\
\text { contact }\end{array}$ & $\begin{array}{l}\text { Spodoptera frugiperda } \\
\text { (Fall armyworm) } \\
\text { (Lepidoptera) }\end{array}$ & $\begin{array}{l}\text { Larvicide (topical: } 0.006 \mu \mathrm{L} \text { EO: }+0.0019 \mu \mathrm{L} \alpha \text {-Cypermethrin } \\
\mathrm{LD}_{50}=0.0000079 \mu \mathrm{L} / \mathrm{mg} \text { of insect; }+0.015 \mu \mathrm{L} \\
\beta \text {-Cypermethrin } \mathrm{LD}_{50}=0.0017 \mu \mathrm{L} / \mathrm{mg} ;+0.0022 \mu \mathrm{L} \\
\text { Fenpropathrin } \mathrm{LD}_{50}=0.000064 \mu \mathrm{L} / \mathrm{mg} ;+0.0011 \mu \mathrm{L} \\
\gamma \text {-Cyhalothrin } \mathrm{LD}_{50}=0.00011 \mu \mathrm{L} / \mathrm{mg} \text { ) (residual: } \\
0.000055 \mu \mathrm{L} \text { EO: }+0.0000016 \alpha \text {-Cypermethrin } \\
\mathrm{LD}_{50}=0.00000021 \mu \mathrm{L} / \mathrm{cm}^{2} ;+0.0000015 \mu \mathrm{L} \\
\beta \text {-Cypermethrin } \mathrm{LD}_{50}=0.0000016 \mu \mathrm{L} / \mathrm{cm}^{2} ;+0.00000062 \\
\text { Fenpropathrin } \mathrm{LD}_{50}=0.00000018 \mu \mathrm{L} / \mathrm{cm}^{2} ;+0.00000019 \mu \mathrm{L} \\
\left.\gamma \text {-Cyhalothrin } \mathrm{LD}_{50}=0.000000033 \mu \mathrm{L} / \mathrm{cm}^{2}\right)\end{array}$ & [29] \\
\hline $\begin{array}{l}\text { Brazil, } \\
\text { Acre }\end{array}$ & Leaves & $\mathrm{SD}$ & $\begin{array}{l}\text { dillapiole } \\
(71.9 \%)+\text { (thiamethoxam/ } \\
\gamma \text {-cyhalothrin, } \gamma \text {-cyhalothrin, } \\
\text { imidacloprid/ } \beta \text {-cyfluthrin, } \\
\beta \text {-cyfluthrin, teflubenzurom/ } \\
\alpha \text {-cypermethrin, } \\
\alpha \text {-cypermethrin) }\end{array}$ & $\begin{array}{l}\text { Topical and } \\
\text { residual } \\
\text { contact }\end{array}$ & $\begin{array}{l}\text { Spodoptera frugiperda } \\
\text { (Fall armyworm) } \\
\text { (Lepidoptera) }\end{array}$ & $\begin{array}{l}\text { Larvicide (topical: } 0.000055 \mu \mathrm{L} \text { EO: }+0.000014 \mu \mathrm{L} \\
\text { Thiamethoxam } / \gamma \text {-Cyhalothrin } \mathrm{LD}_{50}=0.0000016 \mu \mathrm{L} / \text { insect; } \\
+0.00000038 \mu \mathrm{L} \gamma \text {-Cyhalothrin } \\
\mathrm{LD}_{50}=0.000000065 \mu \mathrm{L} / \text { insect; }+0.00016 \mu \mathrm{L} \text { Imidacloprid/ } \\
\beta \text {-Cyfluthrin } \mathrm{LD}_{50}=0.000055 \mu \mathrm{L} / \text { insect; }+0.0000055 \mu \mathrm{L} \\
\beta \text {-Cyfluthrin } \mathrm{LD}_{50}=0.000001 \mu \mathrm{L} / \text { insect; }+0.000011 \mu \mathrm{L} \\
\text { Teflubenzurom } \alpha \text {-Cypermethrin } \\
\mathrm{LD}_{50}=0.0000015 \mu \mathrm{L} / \text { insect; }+0.000012 \mu \mathrm{L} \alpha \text {-Cypermethrin } \\
\mathrm{LD}_{50}=0.0000016 \mu \mathrm{L} / \text { insect) (residual: } 584.85 \mathrm{ppm} \text { EO: } \\
+183.4 \mathrm{ppm} \text { Thiamethoxam } / \gamma \text {-Cyhalothrin LC } \mathrm{LC}_{50}=74.1 \mathrm{ppm} \text {; } \\
+1026.4 \mathrm{ppm} \gamma \text {-Cyhalothrin } \mathrm{LC}_{50}=11.7 \mathrm{ppm}+8455.2 \mathrm{ppm} \\
\text { Imidacloprid } / \beta \text {-Cyfluthrin } \mathrm{LC}_{50}=1512.6 \mathrm{ppm}+927.3 \mathrm{ppm} \\
\beta \text {-Cyfluthrin } \mathrm{LC}_{50}=16.0 \mathrm{ppm} ;+1895 \mathrm{ppm} \\
\text { Teflubenzurom/ } \alpha \text {-Cypermethrin } \mathrm{LC}_{50}=0.8 \mathrm{ppm} ; \\
+206.3 \text { ppm } \alpha \text {-Cypermethrin } \mathrm{LC}_{50}=10.1 \mathrm{ppm} \text { ) }\end{array}$ & {$[28]$} \\
\hline $\begin{array}{l}\text { Brazil, } \\
\text { Acre }\end{array}$ & Leaves & SD & $\begin{array}{l}\text { dillapiole }(71.9 \%)+\text { (profenofos, } \\
\text { fenitrothione, chlorpyrifos, } \\
\text { metomil) }\end{array}$ & $\begin{array}{l}\text { Topical and } \\
\text { residual } \\
\text { contact }\end{array}$ & $\begin{array}{l}\text { Spodoptera frugiperda } \\
\text { (Fall armyworm) } \\
\text { (Lepidoptera) }\end{array}$ & $\begin{array}{l}\text { Larvicide (topical: } 0.000055 \mu \mathrm{L} \text { EO: }+0.000038 \mu \mathrm{L} \text { Profenofos } \\
\mathrm{LD}_{50}=0.0048 \mu \mathrm{L} / \mathrm{mg} \text { of insect; }+0.00045 \mu \mathrm{L} \text { Fenitrothione } \\
\mathrm{LD}_{50}=0.000071 \mu \mathrm{L} / \mathrm{mg} ;+0.000025 \mu \mathrm{L} \text { Chlorpyrifos } \\
\mathrm{LD}_{50}=0.00001 \mu \mathrm{L} / \mathrm{mg} ;+0.0000067 \mu \mathrm{L} \text { Metomil } \\
\left.\mathrm{LD}_{50}=0.0000061 \mu \mathrm{L} / \mathrm{mg}\right)(\text { residual: } 0.006 \mu \mathrm{L} \text { OE: }+0.0068 \\
\text { Profenofos } \mu \mathrm{L} \mathrm{LC} \mathrm{LC}_{50}=0.0013 \mu \mathrm{L} / \mathrm{cm}^{2} ;+0.0017 \mu \mathrm{L} \\
\text { Fenitrothione } \mathrm{LC}_{50}=0.00044 \mu \mathrm{L} / \mathrm{cm}^{2} ;+0.000053 \mu \mathrm{L} \\
\text { Chlorpyrifos } \mathrm{LC}_{50}=0.000016 \mu \mathrm{L} / \mathrm{cm}^{2} ;+0.0083 \mu \mathrm{L} \text { Metomil } \\
\left.\mathrm{LC}_{50}=0.0048 \mu \mathrm{L} / \mathrm{cm}^{2}\right)\end{array}$ & [31] \\
\hline
\end{tabular}

SD: steam-distillation, EO: essential oil, $\mathrm{LC}_{50}$ : lethal concentration for $50 \%, \mathrm{LD}_{50}$ : lethal dose for $50 \%$. 
to the $85.4 \%$ if the EO was used in its totality. Only four experiments did not show the presence of dillapiole: one of them used dried fruits to extract the EO, differing from most of the other experiments that mainly used leaves. Many papers focused on activity against Aedes aegypti, a mosquito vector of several viruses.

Concerning use of the EO, or its main compound in these experiments that isolated it from the rest of the components, a variety of methods were used: topical, with direct application to the insect using a specialized instrument regarding the type or stage of the insect; contact, allowing the insect to move through a surface where the EO was placed, or through the substance diluted in water; fumigation, mostly obtained by evaporation of the solution containing diluted EO; immersion, where the organism was directly submerged in the solution; and spraying. For the fumigation and contact experiments, residual effects were also often determined. Finally, activity was expressed in multiple forms, for instance adulticide, larvicide and ovicide effects, or repellency to oviposition. Almost all results expressed the lethal concentration (LC) of the EO that affected at least $50 \%$ of the insects tested. This concentration was also expressed as lethal dose (LD), lethal time (LT) or effective dose (ED). ED was related to the efficiency of repelling mosquitos from the human body. These measurements depended on how the researcher wished to express EO lethality. Considering for example the larvicidal effect in A. aegypti and where dillapiole was the main compound of the EO applied through contact or fumigation, we find that the $\mathrm{LC}_{50}$ ranged between 57 and $200 \mu \mathrm{g} / \mathrm{mL}$.

Finally, when not specified, we reported only experiments with a lowest duration as there was no significant difference between them: generally, the longer the experiment duration, the lower the concentration needed to obtain the same efficiency.

Piper aduncum EO appears to have promising properties in terms of insecticidal activity as it has been tested in a wide range of different insects and provided positive results. On the other hand, there are many studies that report this property for others EOs, for instance recently described activity of Foeniculum vulgare EO extracts against certain aphid species [75]. These findings support the possibility of discovering suitable substitutes for chemical insecticides.

\section{Acaricidal activity}

Table 3 shows the properties of the EO as an acaricidal and, like in Table 1, we report both classification and scientific name. We were able to collect only five experiments, three of them regarding the effects against Tetranychus urticae, a species of plant-feeding mite that is considered as a pest.

The concentration of dillapiole ranged between $94.84 \%$ and $28 \%$, and in just one of the experiments using plants of $P$. aduncum from Cuba, dillapiole was not present as a component of the EO. The application methods were similar to those used in the insecticide experiments.

There are fewer data on the acaricidal effects, but they still show promise regarding the control of pests in a wide variety of crops and cattle. In this field, we also found many studies on the use of the EOs as acaricides, for instance extracts of Lippia gracilis against Tetranychus urticae [8] or Ocimum gratissimum against Rhipicephalus microplus [95].

\section{Antiparasitic activity}

In Table 4, we report antiparasitic activities, specifying the scientific name of each organism. Articles have been published concerning the toxicity of the EO against various species, such as Haemonchus contortus and Hysterothylacium sp., whereas most of the experiments aimed to determine activity against the genus Leishmania, the protozoa responsible for leishmaniasis, followed by Trypanosoma, which causes Chagas disease. Lastly, a number of studies were related to Plasmodium falciparum, suggesting that it may be more likely to overcome malaria using essential oils against its vector (Table 2), rather than approaches against the protozoa that causes the disease.

When specified, the concentration of dillapiole was always higher than $75 \%$. In this case, the EO was mostly applied through incubation in which it was added to the infected cell cultivation and placed in the best conditions (usually $37{ }^{\circ} \mathrm{C}$ ) for protozoan growth, for at least $24 \mathrm{~h}$. Efficiency was mostly expressed as activity against promastigotes. For instance, in the case of Leishmania, to inhibit the growth of at least $50 \%$ $\left(\mathrm{IC}_{50}\right)$ of promastigotes, about $15 \mu \mathrm{g} / \mathrm{mL}$ of the $\mathrm{EO}$ with dillapiole are needed as the main component.

Antiparasitic activities, mostly antileishmanial effects, represent the second largest group of results, and are supported by other studies showing these properties of various EOs, such as Cryptocarya aschersoniana EO [2] and others.

\section{Synergistic activity}

Table 5 shows the activity of dillapiole in synergy with other chemical substances used as insecticides to determine potential efficacy against Spodoptera frugiperda, a caterpillar known as the fall armyworm, considered a pest due to the damage it causes to a wide variety of crops and the associated economic losses.

In these experiments, two methods of application were used to determine the larvicidal effect: in the first case, they applied the solutions topically to the dorsal side of the larvae, whereas in the second, to verify the residual potential of the synergistic combination, they soaked filter paper in the substance, let it dry and placed it in a Petri dish where the larvae were then situated for $24 \mathrm{~h}$. The concentration of dillapiole was always $71.9 \%$ and one of the chemical compounds that was used by three of the four experiments was cypermethrin and its different forms. Larvicidal activity was systematically tested. These results reinforce the conclusions pointed out by various authors $[5,12]$ concerning synergistic activity.

\section{Conclusions}

Clearly, the Amazon Rain Forest still harbours a huge amount of knowledge. Articles have described the proprieties of just some of the species from this region, and of these, thousands concerned EO properties.

In this review, the authors aim to increase interest in this useful species and its EO, and in a wider context, to highlight 
the possibility of finding natural substitutes for chemical biopesticides.

EOs are a promising natural alternative as insecticides, acaricides and antiparasitic products, but researchers and industry must address several challenges in order to obtain new commercial products. EOs are recognised as ecofriendly, biodegradable and cost effective raw materials [17] and generally present very low mammalian toxicity and short environmental persistence [80]. Unfortunately, precisely due to their short persistence, EOs exert a short duration of action and require several applications or additional efforts on formulation strategies. The most common formulation strategy is to add chemical fixatives or stabilizers to the EO, in order to prolong effects. Fixatives make it possible to slow evaporation of EOs due to their lower volatility in comparison with EOs compounds. Promising results with natural (vanillin) and synthetic fixatives (Glucam P-20, Fixolide) have been reported by Songkro et al. (2012) [98], with several repellent mosquito formulations. The presence of the fixatives was able to improve repellent activity and the longevity of formulation efficacy.

Concerning the mode of action, EOs are generally able to affect insects and mites through neurotoxic effects involving the inhibition of acetylcholinesterase and an effect on the octopamine synapses and GABA receptors. In particular, due to the presence of methylene dioxy rings, dillapiole may inhibit insect P450 cytochrome activity. This mechanism affects the phase I metabolism of xenobiotics, which is responsible for inactivating the insecticides. EOs also exert repellent activity, blocking the odour receptor neurons (ORNs) of the insects $[17,33,80]$.

As reported by Misni et al. (2011) [63], a general approach to the understanding of the insecticidal mode of action of EOs should be similar to that for pyrethroids. After initial excitability, the insects lose the control of their movements and present convulsions, paralysis and ultimately, death.

Dillapiole seems to be the most valuable compound among those forming this EO and for the same reason should be extensively studied. Even though essential oils may be a source of allergy in humans, dillapiole has not been added to the list of 26 fragrance ingredients listed as allergens in Annex III of the European Union's Scientific Committee on Consumer Safety Opinion on Fragrance allergens in cosmetic products (SCCS/1459/11). Interestingly, a study performed by Aciole et al. (2013) [1], on dillapiole identified genetic toxicity on somatic cells of Drosophila melanogaster. Hsuuw and Chan (2015) [44] investigated the effects of dillapiole on mouse oocyte maturation, showing a potential teratogenic effect. The authors suggest that the effect of dillapiole on human oocytes should also be investigated in order to assess the safety of its application as a drug or biocide. These findings indicate that dillapiole should be studied further to determine its possible toxicity in humans. For instance, it would be useful to evaluate its effects in synergy with other compounds to find the most useful match, as it has been shown that the oils are more effective when used in their totality than just in main component form. Other compounds are also of interest based on the data shown in the tables, as in some cases they are even the main compound instead of dillapiole, for example myristicin or 1,8-cineole. Therefore, a more specific study of these isolated components is required to have a wider understanding of the entire EO.

In addition, it is important to define the best way to investigate $P$. aduncum EO or dillapiole in open field studies or infested areas, in order to develop new phytoiatric formulations, such as nanoemulsions or insecticidal sugar baits, which can be successfully adopted as alternative commercial biopesticides. With the same approach, deeper pharmacological investigations that include formulation development should be performed in order to obtain new plant-based antiparasitic drugs.

We also need to understand the consequences of using $P$. aduncum EO as a biopesticide, such as its effect on the environment and its residual phytotoxicity, among others.

The proprieties and effects of this EO must be better analysed and clarified, as the results highlighted in this review are quite promising. Finally, further studies could focus on finding a semisynthetic product from this EO, which would be more economically practical and, at the same time, place lower demands on natural resources.

\section{Conflict of interest}

The authors declare that there are no conflicts of interest.

Acknowledgements. This research was supported by the Universidad Estatal Amazónica, Soil Science department (Ecuador Republic) and the University of Extremadura, Faculty of Sciences (Spain).

\section{Author contribution}

$\mathrm{AD}$ drafted the manuscript and revised the final version. MR participated in the data mining, the literature analysis, and manuscript editing. JBS and TRT provided additional information. TRT contributed to the conception of the research, monitored the study, and revised the final version. All authors reviewed and approved the final version of the manuscript.

Acknowledgements. A.D. was granted by Americampus Program 2019/2020 Junta de Extremadura-Universidad de Extremadura.

\section{References}

1. Aciole EHP, Guimarães NN, Silva AS, Amorim EM, Nunomura SM, Garcia ACL, Cunha KS, Rohde C. 2014. Genetic toxicity of dillapiol and spinosad larvicides in somatic cells of Drosophila melanogaster. Pest Management Science, 70, 559-565.

2. Andrade PM De, Melo DC De, Alcoba AET, Ferreira Júnior WG, Pagotti MC, Magalhães LG, Santos TCL Dos, Crotti AEM, Alves CCF, Miranda MLD. 2018. Chemical composition and evaluation of antileishmanial and cytotoxic activities of the essential oil from leaves of Cryptocarya aschersoniana Mez. (Lauraceae Juss.). Anais da Academia Brasileira de Ciencias, 90, 2671-2678.

3. Araújo MJC, Câmara CAG, Born FS, Moraes MM, Badji CA. 2012. Acaricidal activity and repellency of essential oil from Piper aduncum and its components against Tetranychus urticae. Experimental and Applied Acarology, 57, 139-155.

4. de Araújo MJC, da Câmara CAG, de Souza Born F., Moraes MM de. 2020. Acaricidal activity of binary blends of essential oils and selected constituents against Tetranychus urticae in laboratory/greenhouse experiments and the impact on Neoseiulus californicus. Experimental and Applied Acarology, 80, 423-444. 
5. Belzile AS, Majerus SL, Podeszfinski C, Guillet G, Durst T, Arnason JT. 2000. Dillapiol derivatives as synergists: Structureactivity relationship analysis. Pesticide Biochemistry and Physiology, 66, 33-40.

6. Bennett BC, Baker MA, Gómez Andrade P. 2003. Ethnobotany of the Shuar of Eastern Ecuador. Economic Botany, 57, 145-145.

7. Bernuci KZ, Iwanaga CC, Fernandez-Andrade CMM, Lorenzetti FB, Torres-Santos EC, Faiões VDS, Gonçalves JE, W do Amaral, Deschamps C, Scodro RBDL, Cardoso RF, Baldin VP, Cortez DAG. 2016. Evaluation of chemical composition and antileishmanial and antituberculosis activities of essential oils of Piper species. Molecules, 21, 1698.

8. Born F de S, Camara CAG da, Melo JPR de, Moraes MM de. 2018. Acaricidal property of the essential oil from Lippia gracilis against Tetranychus urticae and a natural enemy, Neoseiulus californicus, under greenhouse conditions. Experimental \& Applied Acarology, 75, 491-502.

9. Brazão MAB, Brazão FV, Maia JGS, Monteiro MC. 2014. Antibacterial activity of the Piper aduncum oil and dillapiole, Its main constituent, against multidrug-resistant strains. Boletin Latinoamericano y del Caribe de Plantas Medicinales y Aromaticas, 13, 517-526.

10. Brogdon WG, McAllister JC. 2005. Insecticide resistance and vector control. Journal of Agromedicine, 9, 327-345.

11. Can Başer KH, Buchbauer G. 2015. Handbook of essential oils: Science, technology, and applications, Second edition. CRC Press: Boca Raton.

12. Carballo-Arce AF, Raina V, Liu S, Liu R, Jackiewicz V, Carranza D, Arnason JT, Durst T. 2019. Potent CYP3A4 inhibitors derived from dillapiol and sesamol. ACS Omega, 4, 10915-10920.

13. Cardoso D, Särkinen T, Alexander S, Amorim AM, Bittrich V, Celis M, Daly DC, Fiaschi P, Funk VA, Giacomin LL, Goldenberg R, Heiden G, Iganci J, Kelloff CL, Knapp S, Lima HC De, Machado AFP, Santos RM Dos, Mello-Silva R, Michelangeli FA, Mitchell J, Moonlight P, Moraes PLR De, Mori SA, Nunes TS, Pennington TD, Pirani JR, Prance GT, Queiroz LP De, Rapini A, Riina R, Rincon CAV, Roque N, Shimizu G, Sobral M, Stehmann JR, Stevens WD, Taylor CM, Trovó M, Van Den Berg C, Werff H Van Der, Viana PL, Zartman CE, Forzza RC. 2017. Amazon plant diversity revealed by a taxonomically verified species list. Proceedings of the National Academy of Sciences of the United States of America, 114, 10695-10700.

14. De Castro CCB, Costa PS, Laktin GT, PHD De Carvalho, Geraldo RB, Moraes J De, Pinto PLS, Couri MRC, Pinto PDF, Silva Filho AA Da. 2015. Cardamonin, a schistosomicidal chalcone from Piper aduncum L. (Piperaceae) that inhibits Schistosoma mansoni ATP diphosphohydrolase. Phytomedicine, 22, 921-928.

15. Ceole LF, Cardoso MDG, Soares MJ. 2017. Nerolidol, the main constituent of Piper aduncum essential oil, has anti-Leishmania braziliensis activity. Parasitology, 144, 1179-1190.

16. Chang W-T, Thissen U, Ehlert KA, Koek MM, Jellema RH, Hankemeier T, van der Greef J, Wang M. 2006. Effects of growth conditions and processing on Rehmannia glutinosa using fingerprint strategy. Planta Medica, 72, 458-467.

17. Chellappandian M, Vasantha-Srinivasan P, Senthil-Nathan S, Karthi S, Thanigaivel A, Ponsankar A, Kalaivani K, Hunter WB. 2018. Botanical essential oils and uses as mosquitocides and repellents against dengue. Environment International, 113, 214-230.

18. Corral ACT, de Queiroz MN, de Andrade-Porto SM, Morey GAM, Chaves FCM, Fernandes VLA, Ono EA, Affonso EG.
2018. Control of Hysterothylacium sp. (Nematoda: Anisakidae) in juvenile pirarucu (Arapaima gigas) by the oral application of essential oil of Piper aduncum. Aquaculture, 494, 37-44.

19. Cossolin JFS, Pereira MJB, Martínez LC, Turchen LM, Fiaz M, Bozdoğan H, Serrão JE. 2019. Cytotoxicity of Piper aduncum (Piperaceae) essential oil in brown stink bug Euschistus heros (Heteroptera: Pentatomidae). Ecotoxicology, 28, 763-770.

20. Costa EV, Teixeira SD, Marques FA, Duarte MCT, Delarmelina C, Pinheiro MLB, Trigo JR, Sales Maia BHLN. 2008. Chemical composition and antimicrobial activity of the essential oils of the Amazon Guatteriopsis species. Phytochemistry, 69, 1895-1899.

21. Costa JGM, Santos PF, Brito SA, Rodrigues FFG. 2010. Composição química e toxicidade de óleos essenciais de espécies de Piper frente a larvas de Aedes aegypti L. (Diptera : Culicidae). Latin America Journal of Pharmacy, 29, 463-467.

22. De La Torre L, Navarrete H, Muriel MP, Maciá M, Balslev H. 2008. Enciclopedia de las plantas útiles en Ecuador. Escuela de Ciencias Biológicas de la Pontífica Universidad Católica del Ecuador \& Herbario AAU del Departamento de Ciencias Biológicas de la Universidad de Aarhus. Quito \& Aarhus: Quito, Ecuador Aahrus, Dinamarca

23. Domingos PRC, Silva Pinto AC da, Santos JMM dos, Rafael MS. 2014. Insecticidal and genotoxic potential of two semi-synthetic derivatives of dillapiole for the control of Aedes (Stegomyia) aegypti (Diptera: Culicidae). Mutation Research - Genetic Toxicology and Environmental Mutagenesis, 772, 42-54.

24. Esperandim VR, Ferreira S, Cristina K, Rezende S, Magalhães LG, Souza JM, Pauletti PM, Januário AH, Laurentz S De, Bastos JK, Símaro GV. 2013. In vitro antiparasitic activity and chemical composition of the essential oil obtained from the fruits of Piper cubeba. Planta Medica, 79, 1653-1655.

25. Farah D, Parisi-Filho R, Magri F, Bonardo V, Fiorino P, Farah V, Fonteles M, Delorenzi J. 2010. Anti-promastigote activity of dillapiole and isodillapiole against Leishmania chagasi. Planta Medica, 45, 1349-1354.

26. Fazolin M, Estrela JLV, Valdomiro C, Lima MS de, Alécio MR. 2005. Toxicidade do óleo de Piper aduncum L. a adultos de Cerotoma tingomarianus Bechyné (Coleoptera: Chrysomelidae). Neotropical Entomology, 34, 485-489.

27. Fazolin M, Estrela JLV, Catani V, Rodrigo Alécio M, Santos de Lima M. 2006. Propiedade inseticida dos óleo essenciais Piper hispidinervum C DC; Piper aduncum L. e Tanaecium nocturnum, (Bur, Rodr.) Shum, K. sobre Tenebrio molitor L. 1758. Ciência e Agrotecnologia, Lavras, 31, 113-120.

28. Fazolin M, Vidal Estrela JL, Medeiros Monteiro AF, Paiva Gomes L, IM Da Silva, Farias Silva MS de. 2016. Potencial sinérgico do óleo de Piper aduncum para inseticidas formulados com misturas de princípios ativos. Revista de Ciências Agrarias Amazon Journal of Agricultural and Environmental Sciences, 59, 362-369.

29. Fazolin M, Vidal Estrela JL, Medeiros Monteiro AF, Silva IM Da, Paiva Gomes L, Farias MS De. 2016. Synergistic potential of dillapiole-rich essential oil with synthetic pyrethroid insecticides against fall armyworm. Ciência Rural, 46, 382-388.

30. Fazolin M, Vidal Estrela JL, Medeiros Monteiro AF, Silva IM Da, Paiva Gomes L, Farias Silva MS De. 2016. Combining the essential oil of Piper aduncum L. with commercial insecticides. Semina: Ciencias Agrarias, 37, 3903-3914.

31. Fazolin M, Vidal Estrela JL, Medeiros Monteiro AF, Silva IM Da, Paiva Gomes L. 2017. Alternative synergistic for acetylcholinesterase inhibitors insecticide. Agro@mbiente On-Line, 11, 232-240. 
32. Ferreira AK, De-Sá-Júnior PL, Pasqualoto KFM, Azevedo RA De, Câmara DAD, Costa AS, Figueiredo CR, Matsuo AL, Massaoka MH, Auada AVV, Kerkis I, Parise Filho R. 2014. Cytotoxic effects of dillapiole on MDA-MB-231 cells involve the induction of apoptosis through the mitochondrial pathway by inducing an oxidative stress while altering the cytoskeleton network. Biochimie, 99, 195-207.

33. Fierascu RC, Fierascu IC, Dinu-Pirvu CE, Fierascu I, Paunescu A. 2020. The application of essential oils as a next-generation of pesticides: Recent developments and future perspectives. Zeitschrift für Naturforschung - Section C Journal of Biosciences, 75, 183-204.

34. da Fonseca Meireles S, Domingos PRC, Silva Pinto AC da, Rafael MS. 2016. Toxic effect and genotoxicity of the semisynthetic derivatives dillapiole ethyl ether and dillapiole n-butyl ether for control of Aedes albopictus (Diptera: Culicidae). Mutation Research - Genetic Toxicology and Environmental Mutagenesis, 807, 1-7.

35. Gaínza YA, Chagas AC de S, Fantatto RR, Chaves FCM, Bizzo HR, Esteves SN. 2016. Piper aduncum against Haemonchus contortus isolates: cross resistance and the research of natural bioactive compounds. Revista Brasileira de Parasitologia Veterinária, 25, 383-393.

36. Garcia FP, Lazarin-Bidóia D, Ueda-Nakamura T, Silva S de O, Nakamura CV. 2013. Eupomatenoid-5 Isolated from leaves of Piper regnellii induces apoptosis in Leishmania amazonensis. Evidence-Based Complementary and Alternative Medicine, 2013, 940531.

37. Guenther E. 1948. The essential oils, Vol. 1. New York: Van Nostrand.

38. Guerrini A, Sacchetti G, Rossi D, Paganetto G, Muzzoli M, Andreotti E, Tognolini M, Maldonado ME, Bruni R. 2009. Bioactivities of Piper aduncum L. and Piper obliquum (Piperaceae) essential oils from Eastern Ecuador. Environmental Toxicology and Pharmacology, 27, 39-48.

39. Hemingway J, Ranson H. 2000. Insecticide resistance in insect vectors of human disease. Annual Review of Entomology, 45, 371-391.

40. Hemingway J, Hawkes NJ, McCarroll L, Ranson H. 2004. The molecular basis of insecticide resistance in mosquitoes. Insect Biochemistry and Molecular Biology, 34, 653-665.

41. Hennessy DR. 1994. The disposition of antiparasitic drugs in relation to the development of resistance by parasites of livestock. Acta Tropica, 56, 125-141.

42. Hidayatulfathi O, Shamsuddin AF, Rajab NF, Nor Zafirah AB, Nur Hazwani AA, Nur Afriza MFO, Lau SY, Nor Azwani MN. 2017. Three repellent gels that contain essential oils from local malaysian plants against dengue vector. Tropical Biomedicine, $34,540-549$

43. Houël E, Gonzalez G, Bessière J, Odonne G, Eparvier V, Deharo E, Stien D. 2015. Therapeutic switching: from antidermatophytic essential oils to new leishmanicidal products. Memórias do Instituto Oswaldo Cruz, 110, 106-113.

44. Hsuuw Y Der, Chan WH. 2015. Apoptotic effects of dillapiole on maturation of mouse oocytes, fertilization and fetal development. Drug and Chemical Toxicology, 38, 469476.

45. Ingaroca S, Castroa A, Norma R. 2019. Composición química y ensayos de actividad antioxidante y del efecto fungistático sobre Candida albicans del aceite esencial de Piper aduncum L. "matico". Revista de la Sociedad Química del Perú, $85,268-279$.

46. Jaramillo-Colorado BE, Duarte-Restrepo E, Nayive PB. 2015. Evaluación de la actividad repelente de aceites esenciales de plantas Piperáceas del departamento de Chocó, Colombia. Revista de Toxicologia, 32, 112-116.
47. Jaramillo MA, Manos PS. 2001. Phylogeny and patterns of floral diversity in the genus Piper (Piperaceae). American Journal of Botany, 88, 706-716.

48. Joshi AS, Li XC, Nimrod AC, elSohly HN, Walker LACA. 2001. Dihydrochalcones from Piper longicaudatum. Planta Medica, 67, 186-188.

49. Krinski D, Foerster LA. 2016. Toxicity of essential oils from leaves of Piperaceae species in rice stalk stink bug eggs, Tibraca limbativentris (Hemiptera: Pentatomidae). Ciencia e Agrotecnologia, 40, 676-687.

50. Krinski D, Foerster LA, Deschamps C. 2018. Ovicidal effect of the essential oils from 18 brazilian Piper species: controlling Anticarsia gemmatalis (Lepidoptera, Erebidae) at the initial stage of development. Acta Scientiarum. Agronomy, 40, e35273.

51. Lainson R, Shaw JJ. 1978. Epidemiology and ecology of leishmaniasis in Latin-America. Nature, 273, 595-600.

52. Leyva M, Marquetti Fernández M, Tacoronte J, Scull R, Tiomno Tiomnova O, Mesa A, Montada D. 2009. Actividad larvicida de aceites esenciales de plantas contra Aedes aegypti (L.) (Diptera: Culicidae). Revista Biomédica, 20, 5-13.

53. Leyva M, Tacoronte J, Marquetti M del C, Montada D. 2008. Actividad insecticida de 3 aceites esenciales de plantas en $\mathrm{Musca}$ domestica (Diptera: Muscidae). Revista Cubana de Medicina Tropical, 60(3). http://scielo.sld.cu/scielo.php?script=sci_arttext\& pid=S0375-07602008000300005.

54. Ling AI, Sulaiman S, Othman H. 2009. Evaluation of Piper aduncum Linn. essential oil (Fam:Piperaceae) against Periplaneta americana (L.). Iranian Journal of Arthropod-Borne Diseases, 3, 1-6.

55. Liu SQ, Scott IM, Pelletier Y, Kramp K, Durst T, Sims SR, Arnason JT. 2014. Dillapiol: a pyrethrum synergist for control of the colorado potato beetle. Journal of Economic Entomology, 107, 797-805.

56. Lopes AA, López SN, Regasini LO, Junior JMB, Ambrósio DL, Kato MJ, da Silva Bolzani V, Cicarelli RMB, Furlan M. 2008. In vitro activity of compounds isolated from Piper crassinervium against Trypanosoma cruzi. Natural Product Research, 22, 1040-1046.

57. Lopes NP, Kato MJ, Andrade EHDA, Maia JGS, Yoshida M, Planchart AR, Katzin AM. 1999. Antimalarial use of volatile oil from leaves of Virola surinamensis (Rol.) Warb. by Waiapi Amazon Indians. Journal of Ethnopharmacology, 67, 313-319.

58. Mamood SNH, Hidayatulfathi O, Budin SB, Ahmad Rohi G, Zulfakar MH. 2017. The formulation of the essential oil of Piper aduncum Linnaeus (Piperales: Piperaceae) increases its efficacy as an insect repellent. Bulletin of Entomological Research, 107, 49-57.

59. Martínez JA, D’Antonino Faroni LR, Soto A. 2013. Porcentaje de perdidada de masa en granos y efecto tóxico del aceite esencial Piper aduncum en Sitophilus zeamais (Coleoptera: Curculionidae). Boletín Científico. Centro de Museos. Museo de Historia Natural, 17, 81-90.

60. Maureen Isabel LS, Maria del Carmen MF, Gisel MM, Oriela PP, Ramón SR, Juan PF, Domingo MD. 2018. Bioactividad de plantas como un método alternativo para el control de mosquitos. Cuba Salud, 2018 [Online].

61. McIntyre KL, Harris CS, Saleem A, Beaulieu L-P, Ta CA, Haddad PS, Arnason JT. 2009. Seasonal phytochemical variation of anti-glycation principles in lowbush blueberry (Vaccinium angustifolium). Planta Medica, 75, 286-292.

62. Miranda Junior RNC. 2010. Avaliação da atividade antiplasmódica in vitro dos óleos de Andiroba (Carapa guianensis Aubl.) e Pimenta-de-macaco (Piper aduncum L). 89 f. Dissertação (Mestrado) - Universidade Federal do Pará, Instituto de Ciências da Saúde, Belém, 2010. Programa de Pós-Graduação em Ciências Farmacêuticas. 
63. Misni N, Othman H, Sulaiman S. 2011. The effect of Piper aduncum Linn. (Family: Piperaceae) essential oil as aerosol spray against Aedes aegypti (L.) and Aedes albopictus Skuse. Tropical Biomedicine, 28, 249-258.

64. Misni N, Sulaiman S, Othman H. 2008. The repellent activity of Piper aduncum linn (Family: Piperaceae) essential oil against Aedes aegypti using human volunteers. Journal of Tropical Medicine \& Parasitology, 31, 63-69.

65. Misni N, Sulaiman S, Othman H, Omar B. 2009. Repellency of essential oil of Piper aduncum against Aedes albopictus in the laboratory. Journal of the American Mosquito Control Association, 25, 442-447.

66. Monzote Fidalgo L, Sariego Ramos I, Montalvo Álvarez AM, Garrido Lorente N, Scull Lizama R, Abreu PJ. 2004. Antipreotozoan properties of essential oils extracted from Cuban plants. Revista Cubana de Medicina Tropical, 56, 230-233.

67. Monzote L, García M, Montalvo AM, Scull R, Miranda M. 2010. Chemistry, cytotoxicity and antileishmanial activity of the essential oil from Piper auritum. Memórias do Instituto Oswaldo Cruz, 105, 168-173.

68. Monzote L, Scull R, Cos P, Setzer W. 2017. Essential oil from Piper aduncum: chemical analysis, antimicrobial assessment, and literature review. Medicines, 4, 49.

69. Navickiene HMD, Morandim ADA, Alécio AC, Regasini LO, Bergamo DCB, Telascrea M, Cavalheiro J, Lopes MN, Bolzani S, Furlan M, Paulista UE, Sp A, Young MCM, De Paulo UDS, Sp SP. 2006. Composition and antifungal activity of essential oils from Piper aduncum, Piper arboreum and Piper tuberculatum. Artigo, 29, 467-470.

70. Oliveira GL, Cardoso SK, Lara Júnior CR, Vieira TM, Guimarães EF, Figueiredo LS, Martins ER, Moreira D de L, Kaplan MAC. 2013. Chemical study and larvicidal activity against Aedes aegypti of essential oil of Piper aduncum L. (Piperaceae). Anais da Academia Brasileira de Ciencias, 85, 1227-1234.

71. Oliveira GL, Vieira TM, Nunes VF, Ruas M de O, Duarted ER, Moreira D de L, Kaplan MAC, , Martins ER. 2014. Chemical composition and efficacy in the egg-hatching inhibition of essential oil of Piper aduncum against Haemonchus contortus from sheep. Brazilian Journal of Pharmacognosy, 24, 288-292.

72. Oliveira JV de, França SM de, Barbosa DR E S, Dutra K de A, Araujo AMN de, Navarro DM do AF. 2017. Fumigation and repellency of essential oils against Callosobruchus maculatus (Coleoptera: Chrysomelidae: Bruchinae) in cowpea. Pesquisa Agropecuaria Brasileira, 52, 10-17.

73. Parise-Filho R, Pasqualoto KFM, Magri FMM, Ferreira AK, BAVG Da Silva, Damião MCFCB, Tavares MT, Azevedo RA, Auada AVV, Polli MC, Brandt CA. 2012. Dillapiole as antileishmanial agent: Discovery, cytotoxic activity and preliminary SAR studies of dillapiole analogues. Archiv Der Pharmazie, 345, 934-944.

74. Parise-Filho R, Pastrello M, Pereira Camerlingo CE, Silva GJ, Agostinho LA, Souza T De, Motter Magri FM, Ribeiro RR, Brandt CA, Carneiro Polli M. 2011. The anti-inflammatory activity of dillapiole and some semisynthetic analogues. Pharmaceutical Biology, 49, 1173-1179.

75. Pavela R. 2018. Essential oils from Foeniculum vulgare Miller as a safe environmental insecticide against the aphid Myzus persicae Sulzer. Environmental Science and Pollution Research, 25, 10904-10910.

76. Pereira ACRL, Oliveira JV de, Gondim Junior MGC, Câmara CAG da. 2008. Atividade inseticida de óleos essenciais e fixos sobre Callosobruchus maculatus (FABR., 1775) (Coleoptera: Bruchidae) em grãos de caupi [Vigna unguiculata (L.) WALP.]. Ciencia e Agrotecnologia, 32, 717-724.
77. Pereira ACRL, Oliveira JV de, Gondim Junior MGC, Câmara CAG da. 2009. Influência do período de armazenamento do caupi [Vigna unguiculata (1.) Walp.], tratado comóleos essenciais e fixos, no controle de Callosobruchus maculatus (Fabricius, 1775) (Coleoptera, Chrysomelidae, Bruchinae). Ciencia e Agrotecnologia, 33, 319-325.

78. Pino O, Sánchez Y, Rodríguez H, Correa TM, Demedio J, Sanabria JL. 2011. Chemical characterization and acaricidal activity of the essential oil from Piper aduncum subsp. ossanum against Varroa destructor. Revista de Protección Vegetal, 26, $52-61$.

79. Rafael MS, Hereira-Rojas WJ, Roper JJ, Nunomura SM, Tadei WP. 2008. Potential control of Aedes aegypti (Diptera: Culicidae) with Piper aduncum L. (Piperaceae) extracts demonstrated by chromosomal biomarkers and toxic effects on interphase nuclei. Genetics and Molecular Research: GMR, 7, 772-781.

80. Regnault-Roger C, Vincent C, Arnason JT. 2012. Essential oils in insect control: Low-risk products in a high-stakes world. Annual Review of Entomology, 57, 405-424.

81. Werka JS, Boehme AK, Setzer WN. 2007. Biological activities of essential oils from Monteverde, Costa Rica. Natural Product Communications, 2, 1215-1219.

82. Rojas-Martínez R, Arrieta J, Cruz-Antonio L, Arrieta-Baez D, Velázquez-Méndez AM, Sánchez-Mendoza ME. 2013. Dillapiole, isolated from Peperomia pellucida, shows gastroprotector activity against ethanol-induced gastric lesions in wistar rats. Molecules, 18, 11327-11337.

83. Salazar LC, Ortiz-Reyes A, Rosero DM, Lobo-Echeverri T. 2020. Dillapiole in Piper holtonii as an inhibitor of the symbiotic fungus Leucoagaricus gongylophorus of leaf-cutting ants. Journal of Chemical Ecology, 46, 668-674.

84. Salehi B, Zakaria ZA, Gyawali R, Ibrahim SA, Rajkovic J, Shinwari ZK, Khan T, Sharifi-Rad J, Ozleyen A, Turkdonmez E, Valussi M, Tumer TB, Monzote Fidalgo L, Martorell M, Setzer WN. 2019. Piper species: A comprehensive review on their phytochemistry, biological activities and applications. Molecules, 24(7), 1364.

85. Sangster N, Batterham P, Chapman HD, Duraisingh M, Jambre L Le, Shirley M, Upcroft J, Upcroft P. 2002. Resistance to antiparasitic drugs: The role of molecular diagnosis. International Journal for Parasitology, 32, 637-653.

86. Sanini C, Massarolli A, Krinski D, Butnariu AR. 2017. Essential oil of spiked pepper, Piper aduncum L. (Piperaceae), for the control of caterpillar soybean looper, Chrysodeixis includens Walker (Lepidoptera: Noctuidae). Revista Brasileira de Botanica, 40, 399-404.

87. Santana HT, Trindade F, Stabeli RG, Silva AAE, Militão JSLT, Facundo VA. 2015. Essential oils of leaves of Piper species display larvicidal activity against the dengue vector, Aedes aegypti (Diptera: Culicidae). Revista Brasileira de Plantas Medicinais, 17, 105-111.

88. Santos TLB Dos, Turchen LM, Dall'oglio EL, Butnariu AR, Pereira MJB. 2017. Phytochemical of Piper essential oil and acute toxicity against Helicoverpa armigera (Lepidoptera: Noctuidae). Revista Brasileirade Ciencias Agrarias, 12, 484-489.

89. Sarrazin SLF, Oliveira RB, Barata LES, Mourão RHV. 2012. Chemical composition and antimicrobial activity of the essential oil of Lippia grandis Schauer (Verbenaceae) from the western Amazon. Food Chemistry, 134, 1474-1478.

90. Scalvenzi L, Radice M, Toma L, Severini F, Boccolini D, Bella A, Guerrini A, Tacchini M, Sacchetti G, Chiurato M, Romi R, Di Luca M. 2019. Larvicidal activity of Ocimum campechianum, Ocotea quixos and Piper aduncum essential oils against Aedes aegypti. Parasite, 26, 23. 
91. Sen R, Chatterjee M. 2011. Plant derived therapeutics for the treatment of Leishmaniasis. Phytomedicine, 18, 1056-1069.

92. Silva WC, Souza Martins JR de, Souza HEM de, Heinzen H, Cesio MV, Mato M, Albrecht F, Azevedo JL de, Barros NM de. 2009. Toxicity of Piper aduncum L. (Piperales: Piperaceae) from the Amazon forest for the cattle tick Rhipicephalus (Boophilus) microplus (Acari: Ixodidae). Veterinary Parasitology, 164, 267-274.

93. Silva JK da, da Trindade R, Alves NS, Figueiredo PL, Maia JGS, Setzer WN. 2017. Essential oils from neotropical Piper species and their biological activities. International Journal of Molecular Sciences, 18, 2571.

94. Silva LS, Mar JM, Azevedo SG, Rabelo MS, Bezerra JA, Campelo PH, Machado MB, Trovati G, Santos AL dos, Fonseca Filho HD, Souza TP de, Sanches TP de. 2019. Encapsulation of Piper aduncum and Piper hispidinervum essential oils in gelatin nanoparticles: a possible sustainable control tool of Aedes aegypti, Tetranychus urticae and Cerataphis lataniae. Journal of the Science of Food and Agriculture, 99, 685-695.

95. Silva Lima A, Milhomem MN, Santos Monteiro O, Arruda ACP, de Castro JAM, Fernandes YML, Maia JGS, Costa-Junior LM. 2018. Seasonal analysis and acaricidal activity of the thymol-type essential oil of Ocimum gratissimum and its major constituents against Rhipicephalus microplus (Acari: Ixodidae). Parasitology Research, 117, 59-65.

96. Silva Pinto AC, Nogueira KL, Maia Chaves FC, Souza da Silva LV, Tadei WP, Pohlit AM. 2012. Adulticidal activity of dillapiol and semi-synthetic derivatives of dillapiol against Aedes aegypti (L.) (Culicidae). Embrapa Amazônia OcidentalArtigo Em Periódico Indexado (ALICE).

97. Soltis PS, Soltis DE, Chase MW. 1999. Angiosperm phylogeny inferred from multiple genes as a tool for comparative biology. Nature, 402, 402-404.

98. Songkro S, Jenboonlap M, Boonprasertpon M, Maneenuan D, Bouking K, Kaewnopparat N. 2012. Effects of Glucam P-20, Vanillin, and Fixolide on mosquito repellency of citronella oil lotions. Journal of Medical Entomology, 49, 672-677.
99. Souto RNP, Harada AY, Andrade EHA, Maia JGS. 2012. Insecticidal activity of Piper essential oils from the Amazon against the fire ant Solenopsis saevissima (Smith) (Hymenoptera: Formicidae). Neotropical Entomology, 41, 510-517.

100. Tebbs MC, Tebbs MC. 1993. Revision of Piper (Piperaceae) in the New World 3. The taxonomy of Piper sections Lepianthes and Radula. Bulletin of the Natural History Museum, 23, 2-51.

101. Tene V, Malagón O, Finzi PV, Vidari G, Armijos C, Zaragoza T. 2007. An ethnobotanical survey of medicinal plants used in Loja and Zamora-Chinchipe, Ecuador. Journal of Ethnopharmacology, 111, 63-81.

102. Tiuman TS, Santos AO, Ueda-Nakamura T, Filho BPD, Nakamura CV. 2011. Recent advances in leishmaniasis treatment. International Journal of Infectious Diseases, 15, e525-e532.

103. Turchen LM, Piton LP, Dall'Oglio EL, Butnariu AR, Pereira MJB. 2016. Toxicity of Piper aduncum (Piperaceae) essential oil against Euschistus heros (F.) (Hemiptera: Pentatomidae) and non-effect on egg parasitoids. Neotropical Entomology, 45, 604-611.

104. Vidal Estrela JL, Fazolin M, Catani V, Lima MS De. 2006. Toxicidade de óleos essenciais de Piper aduncum e Piper hispidinervum em Sitophilus zeamais. Pesquisa Agropecuaria Brasileira, 41, 217-222.

105. Villamizar LH, Cardoso M das G, Andrade J, Teixeira ML, Soares MJ. 2017. Linalool, a Piper aduncum essential oil component, has selective activity against Trypanosoma cruzi trypomastigote forms at $4^{\circ} \mathrm{C}$. Memorias do Instituto Oswaldo Cruz, 112, 131-139.

106. Volpe HX, Fazolin M, Garcia RB, Magnani RF, Barbosa JC, Miranda MP. 2016. Efficacy of essential oil of Piper aduncum against nymphs and adults of Diaphorina citri. Pest Management Science, 72, 1242-1249.

Cite this article as: Durofil A, Radice M, Blanco-Salas J \& Ruiz-Téllez T. 2021. Piper aduncum essential oil: a promising insecticide, acaricide and antiparasitic. A review. Parasite 28, 42.

Reviews, articles and short notes may be submitted. Fields include, but are not limited to: general, medical and veterinary parasitology; morphology, including ultrastructure; parasite systematics, including entomology, acarology, helminthology and protistology, and molecular analyses; molecular biology and biochemistry; immunology of parasitic diseases; host-parasite relationships; ecology and life history of parasites; epidemiology; therapeutics; new diagnostic tools.

All papers in Parasite are published in English. Manuscripts should have a broad interest and must not have been published or submitted elsewhere. No limit is imposed on the length of manuscripts.

Parasite (open-access) continues Parasite (print and online editions, 1994-2012) and Annales de Parasitologie Humaine et Comparée (1923-1993) and is the official journal of the Société Française de Parasitologie. 